\title{
A Clustered and Surrogate-based MDA Use Case for MDO Scenarios in AGILE Project
}

\author{
T. Lefebvre*, N. Bartoli*, S. Dubreuil ${ }^{\dagger}$ \\ ONERA/DTIS, Université de Toulouse, Toulouse, France \\ M. Panzeri ${ }^{\ddagger}$, R. Lombardi \\ NOESIS Solutions N.V., Leuven, Belgium \\ Wim Lammen ${ }^{\S}$ \\ NLR - Netherlands Aerospace Centre, Amsterdam, the Netherlands \\ Mengmeng Zhang ${ }^{\mathrm{I}}$ \\ AIRINNOVA AB, Stockholm, Sweden \\ Imco van Gent"l \\ Faculty of Aerospace Engineering, TU Delft, Delft, The Netherlands \\ P.D. Ciampa** \\ DLR, Air Transportation Systems Institute, Hamburg, Germany
}

In this paper methodological investigations regarding an innovative Multidisciplinary Design and Optimization (MDO) approach for conceptual aircraft design are presented. These research activities are part of the ongoing EU-funded research project AGILE. The next generation of aircraft MDO processes is developed in AGILE, which targets significant reductions in aircraft development cost and time to market, leading to cheaper and greener aircraft solutions. The paper introduces the AGILE project structure and recalls the achievements of the first year of activities where a reference distributed MDO system has been formulated, deployed and applied to the design and optimization of a reference conventional aircraft configuration. Then, investigations conducted in the second year are presented, all aiming at making the complex optimization workflows easier to handle, characterized by a high degree of discipline interdependencies, multi-level processes and multi-partner collaborative engineering activities. The paper focuses on an innovative approach in which knowledge-based engineering and collaborative engineering techniques are used to handle a complex aircraft design workflow. Surrogate models replacing clusters of analysis disciplines have been developed and applied to make workflow execution more efficient. The paper details the different steps of the developed approach to set up and operate this test case, involving a team of aircraft design and surrogate modelling specialists, and taking advantage of the AGILE MDO framework. To validate the approach, different executable workflows were generated automatically and used to efficiently compare different MDO formulations. The use of surrogate models for clusters of design competences have been proved to be efficient approach not only to decrease the computational time and also to benchmark different MDO formulations on a complex optimization problem.

\footnotetext{
*Research Engineer, Information Processing and Systems Department, AIAA Member

${ }^{\dagger}$ Research Engineer, Information Processing and Systems Department

$\doteqdot$ Research Engineer, Research and Innovation Department

${ }^{\S}$ R\&D Engineer Modeling and Simulation, Collaborative Engineering Systems dept.

"I Research Scientist, AIAA Member

"Ph.D. Student, AIAA Student Member

** Research engineer, Integrated Aircraft Design Department, AIAA Member
} 


\section{Nomenclature}

\begin{tabular}{|c|c|c|}
\hline AGILE & $=$ & $\begin{array}{l}\text { Aircraft 3rd Generation MDO for Innovative Collaboration of Heterogeneous Teams of } \\
\text { Experts }\end{array}$ \\
\hline $\mathrm{DC}$ & $=$ & Design Campaign \\
\hline OAD & $=$ & Overall Aircraft Design \\
\hline MDA & $=$ & MultiDisciplinary Analysis \\
\hline MDO & $=$ & Multidisciplinary Design Optimization \\
\hline XDSM & $=$ & eXtended Design Structure Matrix \\
\hline CPACS & $=$ & Common Parametric Aircraft Configuration Schema \\
\hline SEGOMOE & $=$ & Super Efficient Global Optimization based on Mixture Of Experts \\
\hline KADMOS & $=$ & Knowledge- and graph-based Agile Design for Multidisciplinary Optimization System \\
\hline VISTOMS & $=$ & VISualization TOol for MDO Systems \\
\hline SM & $=$ & Surrogate Model \\
\hline DACE & $=$ & Design and Analysis of Computer Experiments \\
\hline POD & $=$ & Proper Orthogonal Decomposition \\
\hline DOE & $=$ & Design Of Experiments \\
\hline LHS & $=$ & Latin Hypercube Sampling \\
\hline PIDO & $=$ & Process Integration and Design Optimization \\
\hline RCE & $=$ & Remote Component Environment \\
\hline IT & $=$ & Information Technology \\
\hline OBS & $=$ & On-Board Systems \\
\hline OEM & $=$ & Operating Empty Mass \\
\hline mFuel & $=$ & Fuel Mass \\
\hline mSystems & $=$ & On-board systems mass \\
\hline MTOM & $=$ & Maximum Take-Off Mass \\
\hline FPG & $=$ & Fundamental Problem Graph \\
\hline $\mathrm{RCG}$ & $=$ & Repository Connectivity Graph \\
\hline MDPG & $=$ & MDAO Data and Process Graph \\
\hline
\end{tabular}

\section{Introduction}

$\mathbf{T}^{\mathrm{N}}$ the last three decades, there has been a growing interest in improving the efficiency of aircraft design processes 1 through the use of multidisciplinary design optimization (MDO) numerical tools and techniques. Nevertheless, the exploitation of the full MDO potential for the development of a complete aircraft is still an open challenge, mainly due to technical and management issues encountered during the set up and operation of such complex architectures. Even though many MDO algorithms have been applied in industrial applications [1, 2], a need for a novel methodology encapsulating disciplinary knowledge and skills to enable the effective management of design projects with ever-increasing design complexities has been identified in the MDO community [3, 4].

Based on that need, since 2015, the EU-funded Horizon 2020 AGILE project is developing the next generation of aircraft MDO processes, focusing on the reduction of aircraft development time in the early stages of the design process and in the context of multi-level and multi-partner collaborative engineering projects. In this paper, the investigation of an innovative approach to set up complex workflows is presented. This approach was aimed at simplifying complex workflows by clustering a set of design competences and using surrogate models to represent those clusters. An overview of the AGILE project structure and its main past achievements in the first half of the project will be presented in Section III Then, the different scenarios investigated will be detailed in Section III These scenarios were aimed at building multidisciplinary processes based on surrogate models in order to compare several MDO strategies. The

\footnotetext{
*http://www.agile-project.eu accessed: May 17th 2018
} 
adaption of the aircraft design workflow is described in Section $[\mathrm{IV}]$ with a focus on the different steps of the retained approach. Finally, different execution workflows are generated and used to compare different MDO formulations in Section $\nabla$

\section{AGILE project overview}

In AGILE, a new paradigm and software framework are under development [5, 6] that will enable the efficient collaboration among international multi-site aircraft design teams. The AGILE project is structured into three sequential phases, targeting design campaigns with increasing levels of complexity, addressing different aircraft configurations and MDO techniques. The overall structure is shown in Figure 1

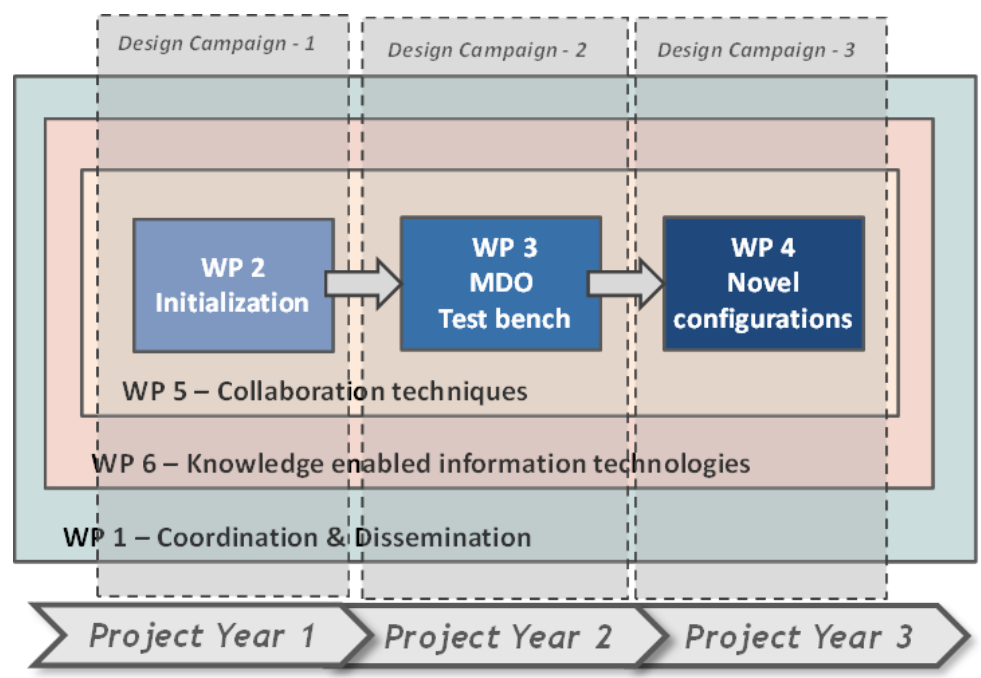

Fig. 1 AGILE project structure

In the first phase (Initialization), a reference aircraft configuration was optimized using state-of-the-art techniques. The reference MDO problem was then used to investigate and benchmark novel optimization techniques individually and later in smart combinations (MDO Test bench). Finally, the most successful MDO strategies are applied to significantly different aircraft configurations (Novel Configurations). The three sequential work packages are embedded within two enabling layers. The first enabling layer (Collaboration techniques) targets the development of the technologies enabling distributed collaboration, comprising the process of collaboration between involved specialists, collaborative pre- and post-processing, visualization and the enhancement of existing framework. The second enabling layer (Knowledge enabled technologies) develops the information technologies, which support the management and the formalization of knowledge within an MDO process. The parallel activities are clustered in three phases (or periods), Design Campaigns (DC), each one lasting one year. Each of the sequential design campaigns focus on the solution of the use cases, which are set up to develop specific collaborative and knowledge-based technologies. DCs address an increasing complexity from use case perspective (progressing from conventional aircraft to novel configurations), and from MDO environment perspective (from the state-of-the-art MDO system to the third generation system).

\section{A. Design campaign 1}

The DC-1 is the first use case in the project that has been formulated and collaboratively solved by the AGILE team. This case consisted of the design and optimization task for a large regional jet, with Entry Into Service 2020. Starting from the specification of the Top Level Aircraft Requirements provided by the aircraft manufacturer partner (Bombardier), an Overall Aircraft Design (OAD) task targeting conceptual and preliminary development stages was implemented in DC-1. Figure 2 shows a representation of the DC-1 distributed OAD process. The figure indicates the domains of the specialists' competences which have been integrated into the process, the location where such simulation competences are hosted, and the specific partners providing such a competence within their IT networks. The corresponding deployed collaborative MDO workflow is represented in Figure 3 A design exploration method was "calling" the OAD process (here labelled as MDA) as a remote service, which integrated all the distributed disciplinary 


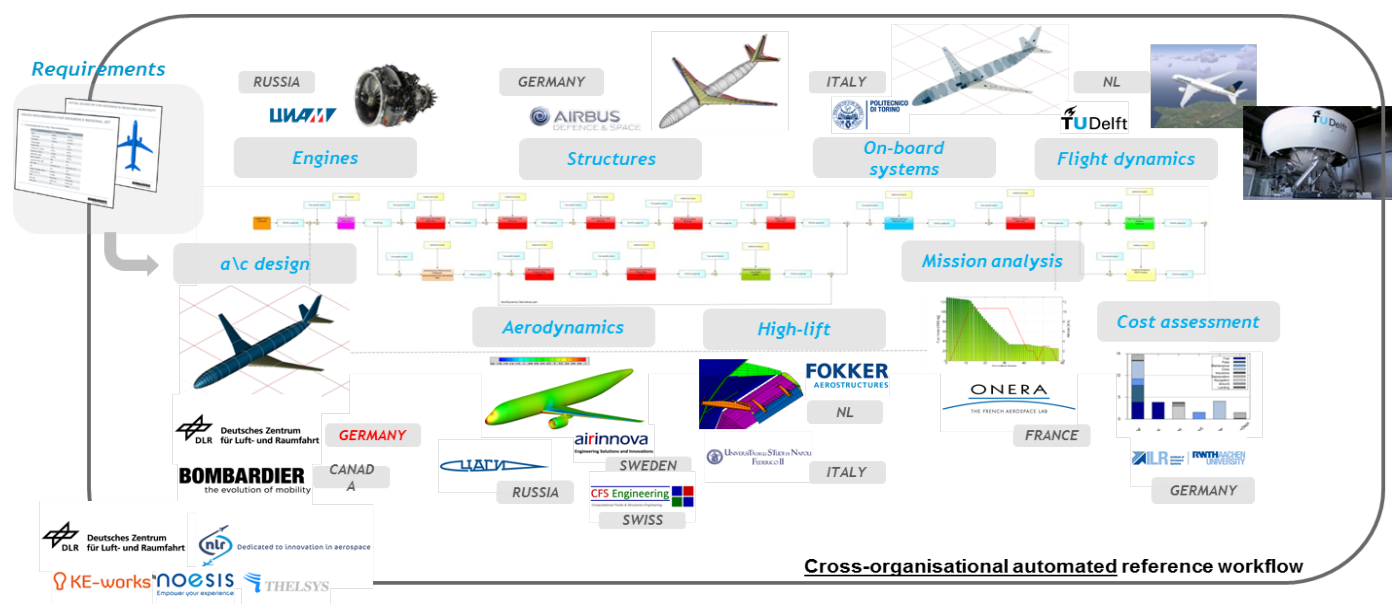

Fig. 2 AGILE Collaborative design process: individual competences are distributed multi-site, and hosted at the different partners' networks

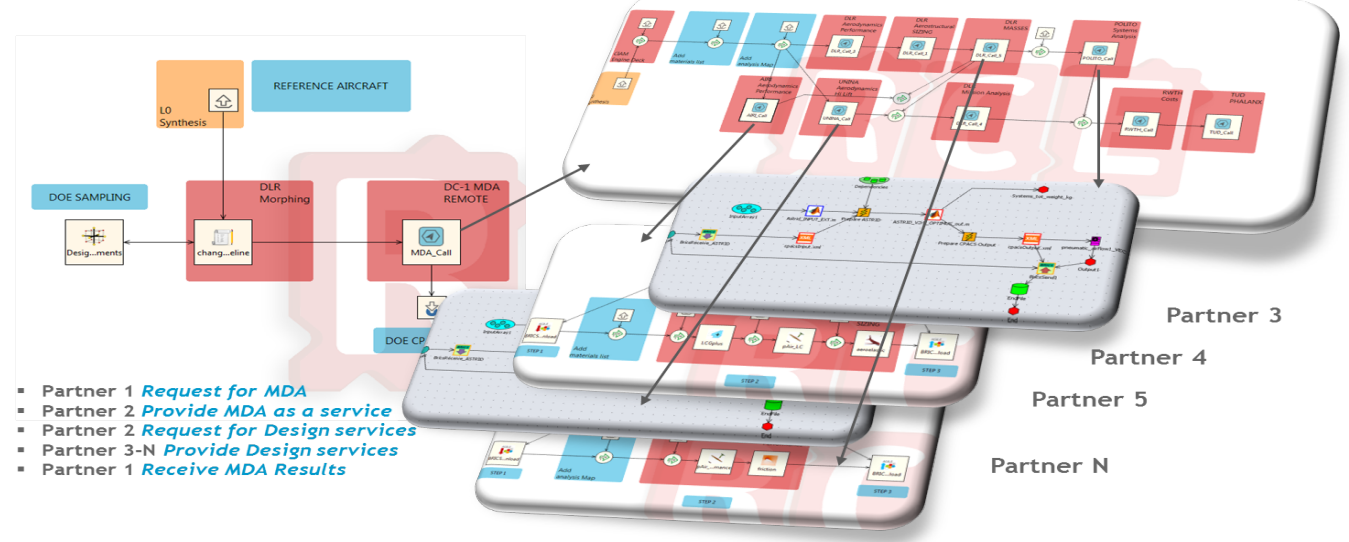

Fig. 3 AGILE DC-1 workflow. Partner 1 deploys a Design Of Experiment requesting as remote service the cross-organizational MDA workflow, deployed at Partner 2 . The MDA is composed by disciplinary competences provided as remote services to Partner 2 by Partners 4 to $\mathrm{N}$. 
competences, which were in turn called as remote services (deployed as disciplinary workflows) within the MDA process. All competences communicated via a CPACS 7 model corresponding to the AGILE aircraft product model. They were deployed as disciplinary workflows and provided as remote services. Furthermore, the deployed "workflow of workflows" has been provided as "service of services" and coupled to a surrogate based optimization strategy, named SEGOMOE, developed by ONERA [7]. An MDO problem was therefore formulated for the optimization of the reference aircraft using an MDF formulation resulting in an improved design.

\section{B. Design campaign 2}

The DC-2 activities were based on the DC-1 work, and were implemented during the second year of the project. In addition, the number of use cases was expanded to five parallel ones. For each use case, a novel MDO strategy (addressing a specific collaborative scenario) was investigated and assessed for the resolution of the design of the reference aircraft. Depending on the test cases, classical MDO formulations (such as MDF, IDF [8] or Analytical Target Cascading [9]) or more adapted ones have been proposed. The five use cases were:

1) Use case focused on the improvement of MDO strategies with the development and integration of new design competences in terms of optimization algorithms and surrogates modelling. These investigations are presented in [10, 11].

2) The implementation of Uncertainty Quantification (UQ) methods and robust based design optimization in complex, variable fidelity optimization was the objective of the second use case [10].

3) The development of mixed-fidelity MDO strategy was tackled with the integration of high-fidelity design competences and its combination with Overall Aircraft Design (OAD) level. The process is presented in [12] and illustrated on Figure 4 a.

4) A multi-scale application is described in [13] aiming at investigating the improvement of involving an aircraft component supplier (aircraft rudder) in the overall aircraft optimization process while keeping its specific framework. The coupled optimization problem is illustrated on Figure 4 b.

5) A large-scale system-of-systems application was also studied, coupling Aircraft - Engine - On-board systems (OBS) - Emissions in a distributed framework approach with the involvement of disciplinary services from the other partners. [14]

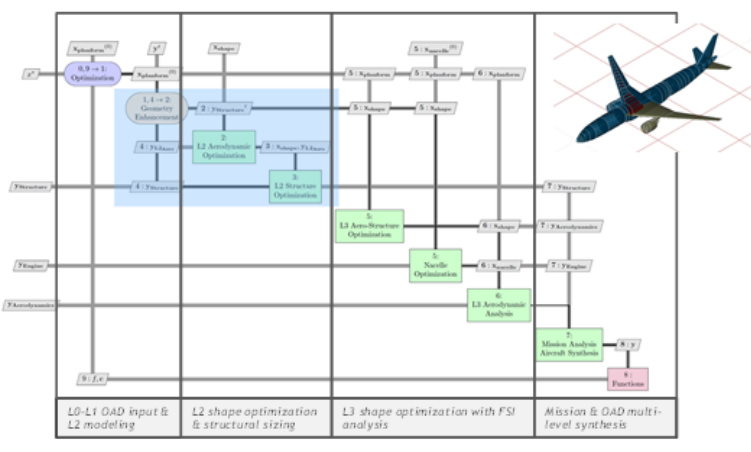

(a) Hi-Fi multi-level optimization formulation

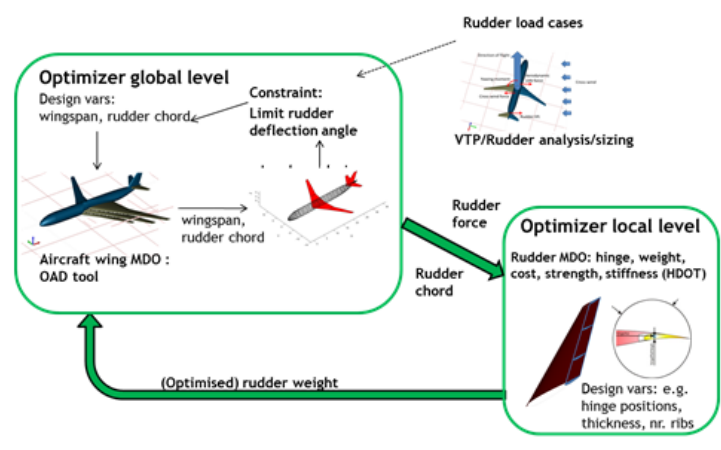

(b) Rudder optimization

Fig. 4 DC-2 investigation examples

Furthermore, based on best practices developed in DC-1, during the DC-2 the overall AGILE framework was enhanced by knowledge-based technologies [15] and IT solutions [16], which contributed to an acceleration of the deployment of the complex MDO processes addressed by the DC-2 use cases. This paper will present DC-2 investigations performed on a use case focusing on enhanced MDO strategies which took advantage of surrogate models to converge the optimization process more rapidly.

\footnotetext{
http://www. cpacs.de - A Common Language for Aircraft Design, accessed: August 28th 2017
} 


\section{MDO through surrogates}

All the methods developed during DC-2 have the common goal of enhancing the optimization of complex workflows, which are characterized by a high degree of discipline interdependencies, high number of design variables in the context of multi-level and multi-partner collaborative engineering projects. One of the most straightforward solutions is the use of surrogate models [17]. A surrogate model (SM) is an analytical formula that replaces a complex model, or even a design analysis workflow, by means of data fitting. Consequently a surrogate model requires only little computation time, which is particularly useful for capturing complex analysis methods and applying them multiple times as part of a global optimization. In collaborative design studies during the early aircraft design phases, surrogate models are valuable to support the collaborative analysis of as many design alternatives as possible in a short time and at low cost, preferably with as much knowledge of the systems under consideration as possible.

\section{A. Surrogate model scenarios}

In the context of DC-2, two main scenarios were considered for the use of surrogate models to enhance MDO strategies. The first scenario (see Figure 5) is related to the investigations of MDO formulations on complex workflows. The objective is to benchmark various MDO formulations such as MultiDisciplinary Feasible (MDF), Individual Discipline Feasible (IDF), Collaborative Optimization (CO). All of these different formulations are described in [8]. In order to compare these formulations in terms of number of function evaluations and/or accuracy of the optimal solution, the idea is to take advantage of surrogate models to reduce the computational costs. The key point here is to use surrogate models, instead of real tools while keeping the disciplinary results accurate. The accuracy of the surrogate models (computed for instance with the Root Mean Square Error criterion on a validation set of points) can be reduced with the use of a large database or with an iterative process to enrich the database as described in [18--20].

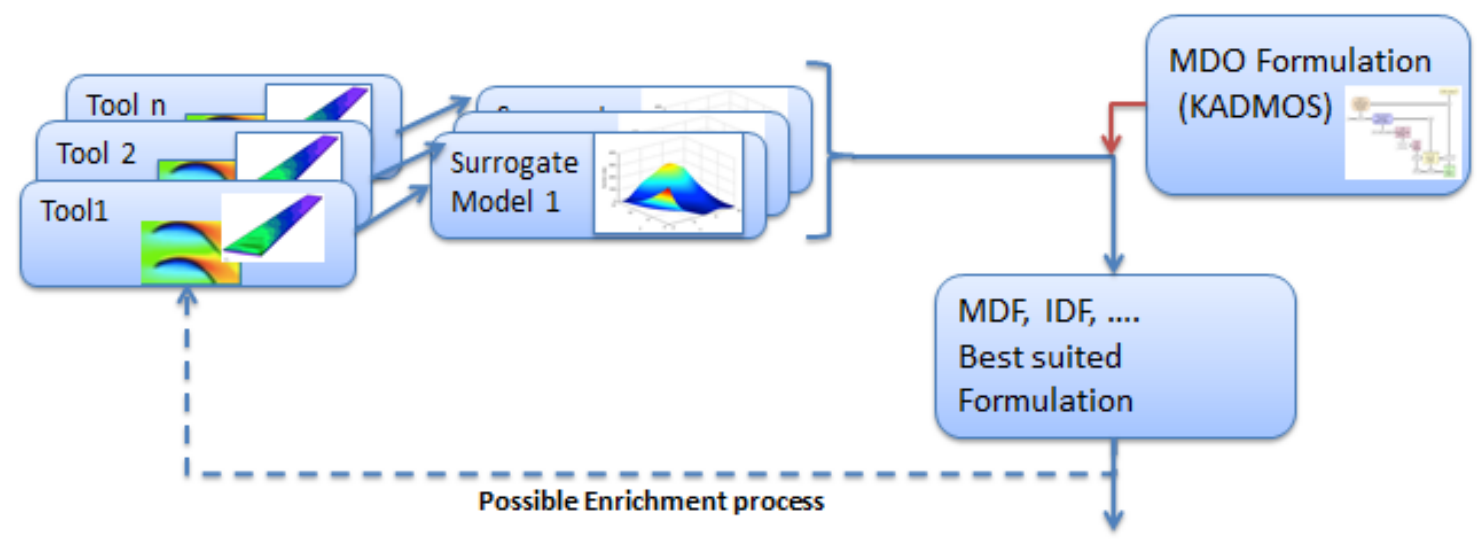

Fig. 5 Scenario for automatic MDO process

The second scenario (see Figure 6 concerns the optimization process using an adaptive construction of disciplinary surrogate models and the propagation of uncertainty associated to each surrogate. By using surrogate models in an MDO process instead of the real tools, some approximation errors are done and they are propagated within the process. In the Efficient Global MDO strategy (EGMDO - see [21]), each disciplinary solver is replaced by a surrogate model (a Gaussian process), allowing to use the mean value and the associated modelling uncertainty in the search of the optimum. This is achieved by propagating the uncertainties introduced by the use of the surrogate models to the objective function. This uncertainty propagation is followed by an iterative process seeking to enrich the surrogate models by calling the disciplinary solvers at some well-chosen points in order to increase their accuracy in the vicinity of the optimum. This strategy mainly relies on the development of a new random field approximation based on polynomial chaos expansion and Gaussian process interpolation[22].

The paper will focus on the different steps considered to set up and operate a specific use case related to the first scenario. 


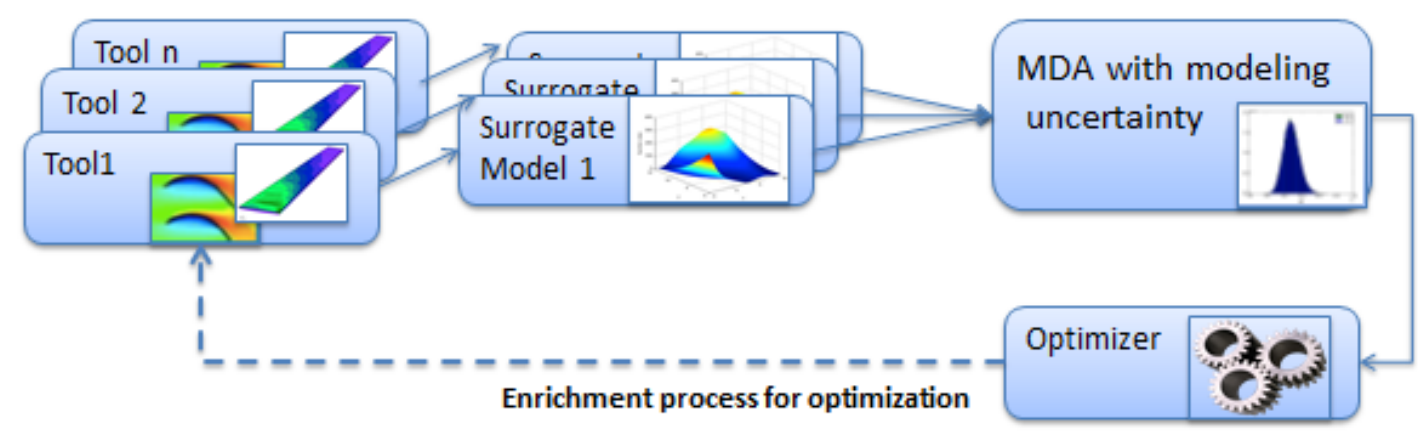

Fig. 6 Scenario relative to propagation of the modeling uncertainty in the process

\section{B. Collaborative architecture}

In AGILE, the MDA/MDO workflows are configured, deployed and executed by making use of PIDO (Process Integration and Design Optimization) environments available at the different process integration sites. Multiple PIDO environments are available in AGILE. One integration environment used in AGILE is the "Remote Component Environment" (RCE) by means of Optimus[23]. Both are deployed in AGILE to compose the main processes, as well as disciplinary sub-processes. The cross-organizational mechanism available in AGILE is Brics[24], developed by NLR. Brics provides technology for interconnecting PIDO environments. It comprises a protocol and supporting middleware for creating cross-organisation workflows as federations of native and legacy local workflows, tools and scripts, complying with the prevailing security constraints. Therefore, nested complex collaborative MDO workflows, connecting multiple organizations, can be deployed. Thanks to the standardized interface by means of CPACS, processes implemented using different PIDO platforms can be integrated in the same MDO. A schematic of workflows in different administrative domains is illustrated in Figure 7. More information on all the developments of collaborative architecture is available in [15, 25].

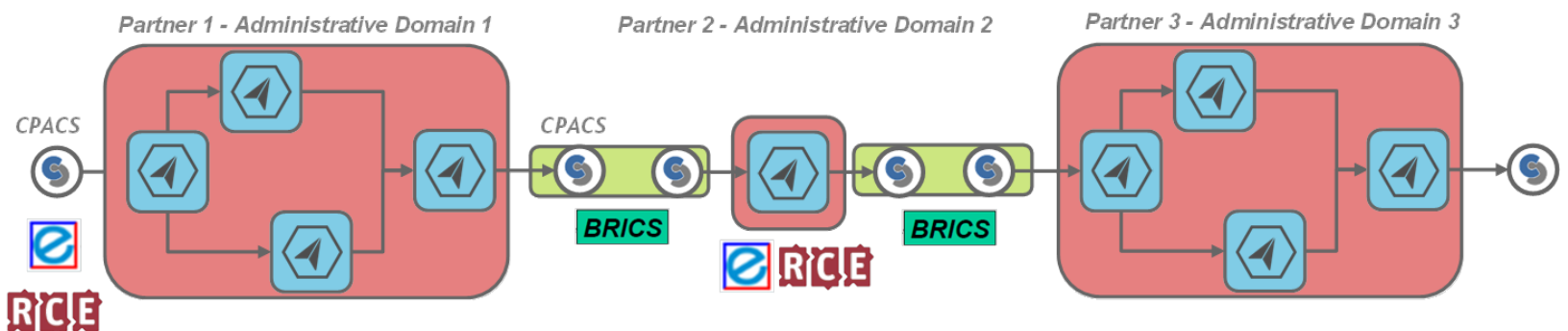

Fig. 7 Connection of PIDO workflows hosted at multiple administrative domains

\section{Knowledge architecture}

The knowledge architecture under development in AGILE integrates different applications to enhance the MDO development process. The full AGILE knowledge architecture is discussed in [16]. Here, three elements of the architecture have been used to support the creation of the different surrogate models and optimization workflows: the graph-based MDO formulation system KADMOS (Knowledge- and graph-based Agile Design for Multidisciplinary Optimization System)[26], the visualization tool for MDO systems VISTOMS (VISualization TOol for MDO Systems)[27], and the data standard to store multidisciplinary system formulations CMDOWS (Common MDO Workflow Schema)[28].

The five main stages of the AGILE development framework are shown in Figure 8. KADMOS and VISTOMS support the development process in the formulation phase of the framework and enable the design team to formulate MDO systems of any size and complexity. This support is provided by KADMOS using a graph-theoretic approach for

http://rcenvironment.de accessed May 17th 2018 


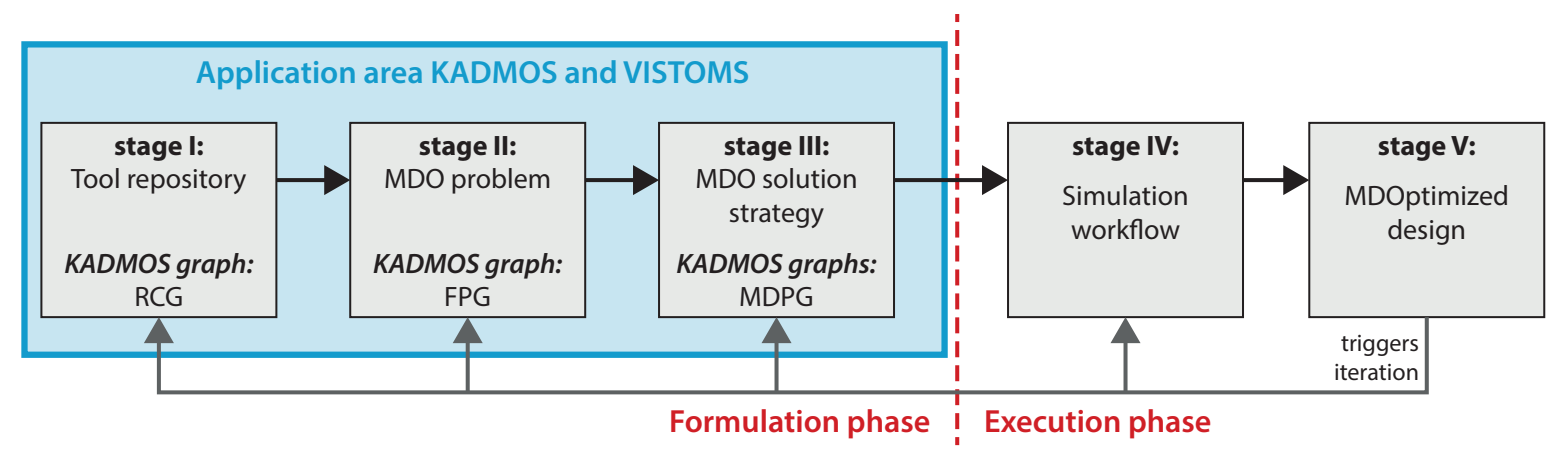

Fig. 8 The five stages of a multidisciplinary system in the AGILE development framework

the setup and manipulation of multidisciplinary systems. In this approach, different graphs are created to represent the three different formulation stages shown in Figure 8

Repository Connectivity Graph (RCG): The RCG is a graph that represents the repository of (CPACS-compatible) tools that are available to the design team. CPACS-compatible tools all operate on a CPACS input file and create a CPACS output file. KADMOS interprets these different files and establishes the interdisciplinary dependencies (couplings), system inputs and system outputs.

Fundamental Problem Graph (FPG): Based on the RCG an FPG can be created by the design team. This FPG is an enriched subgraph of the RCG, hence only a selection of the RCG tools which are necessary to solve a certain problem are still in the graph. Furthermore, key variables are indicated in the FPG, such as design variables and quantities of interest, which are necessary to define an MDO solution strategy. In this paper, the FPG is used to define different clusters for which Designs of Experiments (DOE) are executed to be able to create the surrogate models of a subset of disciplinary tools. In addition, FPGs are also used to define optimization problems that can then be solved based on strategies such as MDF and IDF.

MDAO Data and Process Graph (MDPG): The MDPGs are automatically created by KADMOS based on the FPG. If the FPG contains a definition of a DOE strategy for a cluster of tools, then the MDPG will contain the description of the data and process flow required to execute this DOE. The MDPG itself is still just a combination of two graphs in KADMOS and cannot be executed.

The different KADMOS graphs can be stored as XML files using the CMDOWS definition. These CMDOWS files enable a link between the formulation and execution of the MDO system. The graphs of the final formulation stage (stage III: MDO solution strategy) can be opened by PIDO software that have CMDOWS parsers to build executable workflows based on the CMDOWS definition.

As the graphs grow in size very quickly, their visualization becomes a challenge, while at the same time this would help the design team to inspect and debug the multidisciplinary system in each stage of the process. In AGILE, VISTOMS has been developed for this purpose and it is used in this paper to visualize the different KADMOS graphs. Throughout this paper the dynamic eXtended Design Structure Matrix (XDSM)[29] view from VISTOMS is used to represent the different KADMOS graphs.

\section{Application to aircraft design}

The scenario 1 described in Section III.A should demonstrate the improvements brought by the use of surrogate models on the optimization of complex workflows in the context of multi-level and multi-partner collaborative projects. A typical application of these investigations is the former MDA workflow defined and implemented during DC-1 activities with a realistic complexity of the problem w.r.t. industrial aircraft design (in terms of number of design competences, amount of coupling ...). The regional jet aircraft configuration studied during DC-1 is depicted on Figure 9 and Figure 10 provides an overview of the corresponding MDA in XDSM format.

The objective was therefore the preparation of the workflows for the envisaged scenarios, using the DC-1 MDA as use case. The different steps required to build the "MDA through surrogates" process are the following:

- Identify the disciplinary tools and their associated domain of variation for each of the inputs.

- Create the associated DOEs and build the associated surrogate models.

- Build the associated workflow within any PIDO framework. 


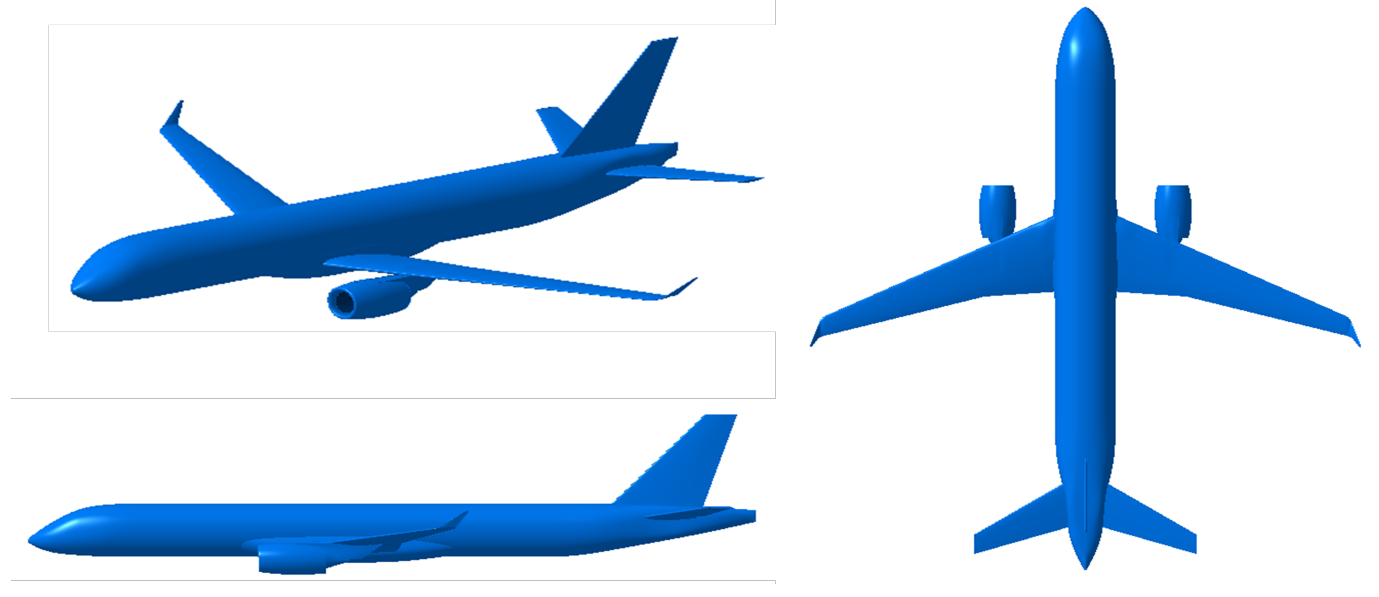

Fig. 9 Collaborative DOE study: nested steps approach

- Run the scenarios.

\section{A. DC-1 simplification}

Figure 10 presents the full DC-1 MDA process. A first analysis indicated that more than 2000 connections can exist between design competences and that some design competences have more than one hundred inputs and outputs. In order to reduce the complexity of the problem while keeping as much as possible its similarity w.r.t aircraft design process, some design competences, like Flight Dynamics assessment and Cost assessment, were removed as they were only used as post processing tools in the workflow. In addition, the engine characteristics are fixed. The retained design competences from DC-1 MDA, mainly made of low to medium fidelity tools, are the following:

- Aerodynamics performance provided by DLR (German Aerospace Center)

- High Lift Performance provided by UNINA (University of Naples "Federico II")

- Propulsion system performance provided by CIAM (Central Institute of Aviation Motors)

- Loads and structural sizing provided by DLR

- On-board systems design provided by POLITO (Politecnico di Torino)

- Mission performance provided by DLR.

All these modifications were not sufficient to reduce the dimensions of the coupling variables (ie connections between the design competences) that would prevent the use of surrogates' capabilities. Indeed the main difficulty to build a SM is driven by the input characteristics: their number and their location. Two main sources of high number of coupling variables can be identified as follows:

1) The coupling between the Geometry and most of the other design competences, such as Aerodynamic performance one, need as inputs, the whole geometry of the aircraft, stored in multiple CPACS branches.

2) The coupling between the Aerodynamic performances and the Mission performance as the Mission performance tools need, as inputs, the whole aerodynamic performance map, stored in a CPACS branch. This lookup table of aerodynamic coefficients is given as function of the mission dependent parameters Mach, Reynolds Number (Re), Angle of Yaw (AoY) and Angle of Attack (AoA).

In order to reduce drastically the $1^{\text {st }}$ source of coupling, a design competence was introduced in the workflow: the Aircraft Morphing design competence (provided by DLR) that enables the modification of wing geometry from a set of design parameters which are not explicitly defined/directly accessible in CPACS. Therefore the full wing geometry, representing hundreds of variables can be controlled by less than a dozen parameters. This design competence was already used for the MDO application of DC-1 as a pre-processing tool and is now introduced inside the workflow. For the $2^{\text {nd }}$ source of coupling, another approach was retained through the use of a specific surrogate model that should embed the Aerodynamic look up table and that is described more in detail in the next paragraph. 


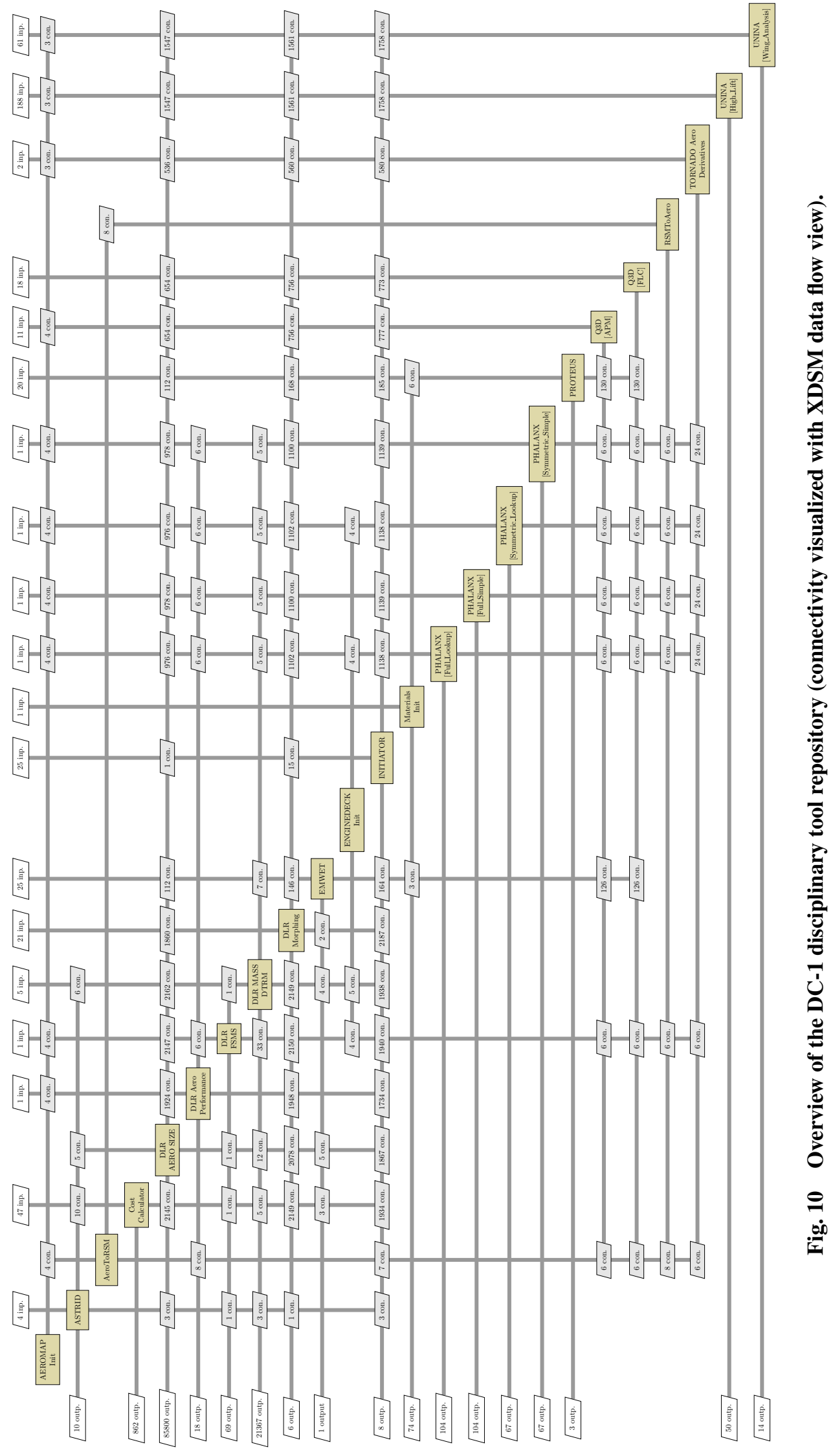




\section{B. Clustering of design competences}

Adding new tools, such as Aircraft Morphing Tool in the workflow, will only lead to reduce the coupling if it is clustered with the other design competences. For instance, making a cluster of the Morphing tool and a Structural sizing tool will expose a limited set of inputs (here the wing design parameters) of the cluster and a limited set of outputs (here the wing weight) as outputs, while keeping the full geometry description as an internal coupling variables between the clustered tools. As an extension of this approach, it was decided to make clusters of design competences of the MDA exposing the following characteristics.

- These clusters should exhibit low dimensions of inputs (less than 20) in order to be accurately represented by a surrogate model.

- These clusters should not have internal feedback coupling between design competences (to prevent the use of convergence process inside the cluster).

- These clusters should be representative of an aircraft design process.

In order to fulfill those requirements, four clusters were built using the retained design competences.

Aerodynamic Cluster This cluster gathers Morphing tool and aerodynamic performance computations including the low speed configurations. It takes as input the wing design parameters and provides the lookup tables for aerodynamic coefficients, related to the specified wing design.

On-board systems Cluster This cluster aims at providing the On Board systems performance, in terms of weights and power, using the wing design parameters and other inputs such as the Fuel Weight and other operational weights such as MTOM (Maximum Take-Off Mass).

Structural sizing and Weight Cluster This cluster provides the weight breakdown of the whole aircraft, using as inputs the wing design parameters, the fuel weight and the systems weight. It also contains the Load and structural sizing competence that sizes the wing structure and computes its weight.

Mission performance Cluster This cluster contains the Mission performance tool and uses as inputs, the wing design parameters, the operational weights and the Aerodynamic look up tables to run the full mission and provides the fuel weight.

Figure 11 provides the enriched graph [16, 27] of the four clusters. One can notice that, each cluster can contain design competences of various partners that will be called through AGILE framework.

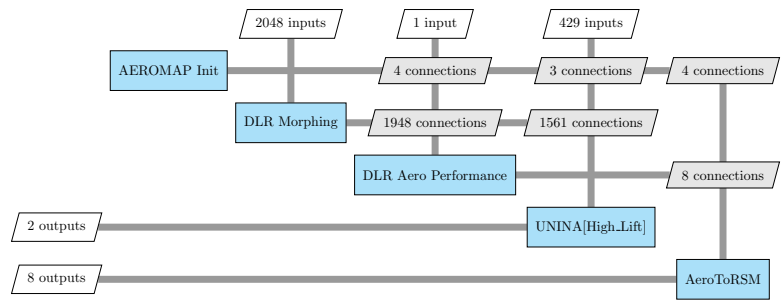

(a) Graph of Aero Cluster

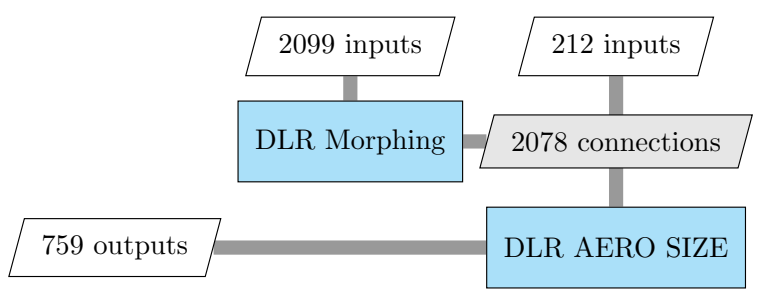

(c) Graph of Weight Cluster

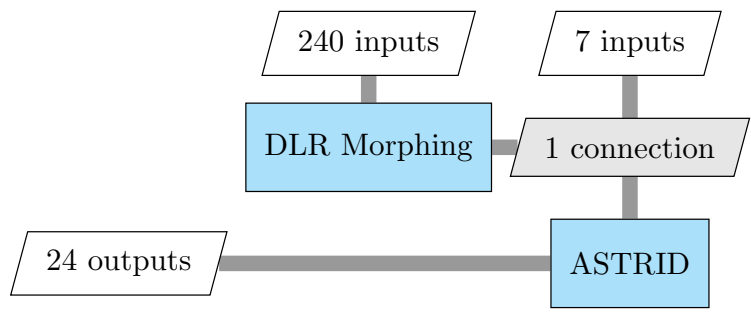

(b) Graph of System Cluster

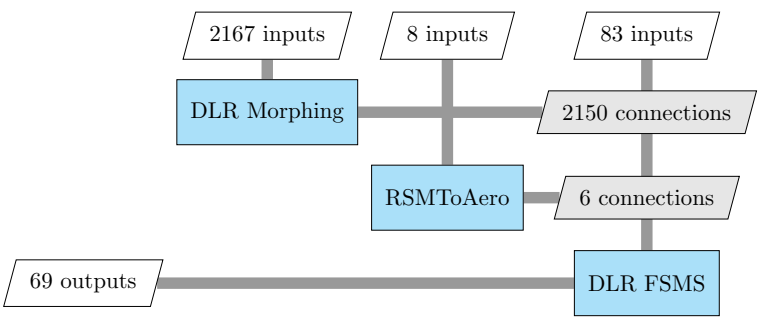

(d) Graph of Mission Cluster

Fig. 11 Graphs of the 4 retained clusters (two-step approach)

At this step, two approaches have been identified in order to derive a surrogate model of the Aero Cluster and link it to the Mission Cluster.

- A two-step approach: In this case the AeroCluster SM predicts - as a function of wing design parameters - a 
representation of the aero lookup tables, e.g. by predicting polynomial coefficients that will be transferred to the MissionCluster SM.

- A "all-in-one" approach: In this case the AeroCluster SM directly predicts the aerodynamic coefficients as a function of wing design parameters and mission parameters (Mach, Re, AoY and AoA). As such the AeroCluster SM becomes an integrated part of the MissionCluster (and therefore also of the MissionCluster SM which has to be derived).

Eventually, Figure 12 summarizes the MDA workflows obtained with the clusters defined above for the two possible approaches. In agreement with the clusters' requirements, both MDA are representative of the DC-1 aircraft design problem with different disciplines (Aerodynamic, Structure, Performance) coupled together. A surrogate model of each cluster now needs to be created in order to build the MDA workflow.

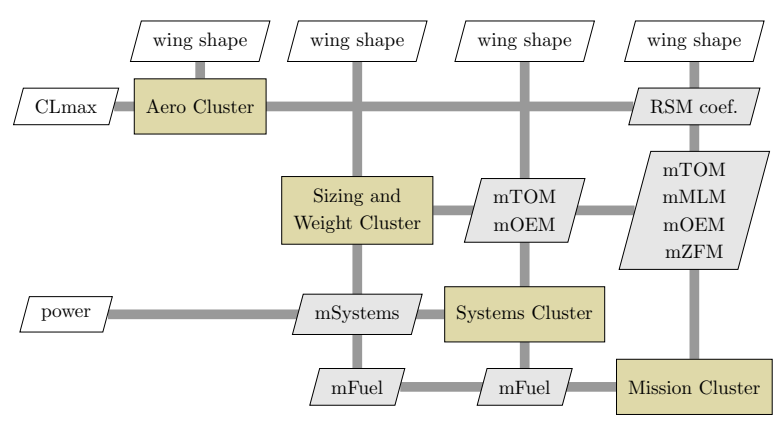

(a) two-step approach

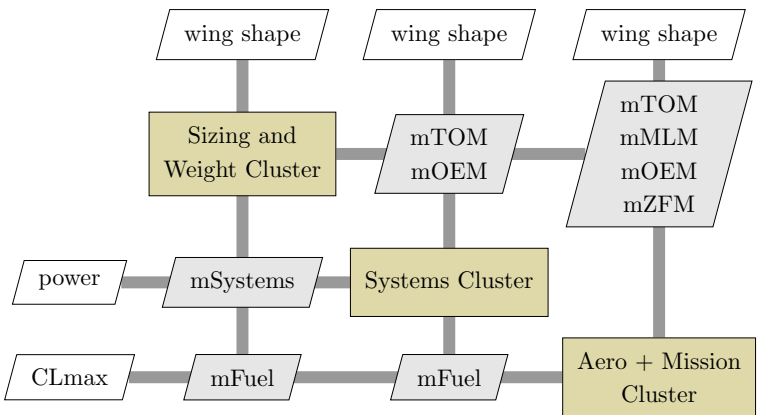

(b) "all-in-one" approach

Fig. 12 Workflow(s) of MDA through surrogates with the two different approaches

\section{Results}

\section{A. DOE}

After the formulation of design competence clusters, they have been implemented as collaborative service oriented workflows, and executed within DOE studies in order to generate the databases for the clusters' surrogate models. An XDSM view for each of the clusters DOEs, automatically created by KADMOS[16] (i.e. the MDAO Data and Process Graphs for the two-step approach) is provided in Figure 13. Each DOE study only exposes from 7 to 11 independent variables, including wing design parameters (7) and aircraft masses (e.g. Operating Empty Mass (OEM)) as coupling variables ( 0 to 4$)$ that will be provided by the other clusters.

The DOE sampling plans have been generated for each cluster, by using a LHS (Latin Hypercube Sampling) sampling method. The number of DOE samples was selected to minimize the numbers of calls to the cluster while providing a sufficient accuracy. Thereafter, the four clusters have been integrated and executed as collaborative workflows. The characteristics of the DOE sampling plan are given in Table 1 .

Table 1 Characteristics of cluster DOE studies database

\begin{tabular}{l|c|c} 
& Initial DOE samples & Number of design variables \\
\hline Aero Cluster & 40 & 7 \\
\hline System Cluster & 60 & 10 \\
\hline Weight Cluster & 60 & 10 \\
\hline Mission Cluster & 70 & 11
\end{tabular}

A collaborative DOE study service approach has been developed within the DC-2 by DLR with the objective to facilitate the execution of collaborative DOE studies, whose different steps are performed at different organizations.

The nested steps are illustrated in Figure 14 and briefly addressed in the following. 


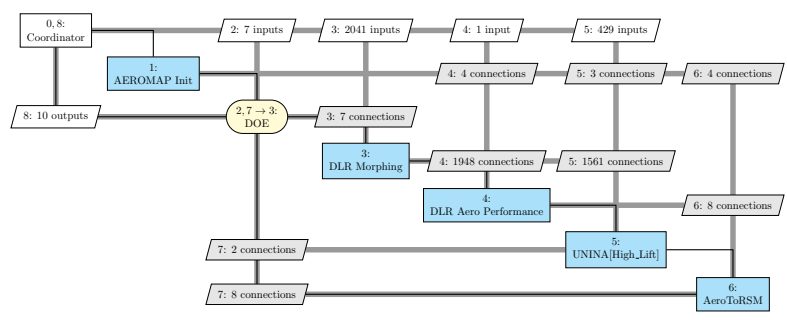

(a) DOE of Aero Cluster

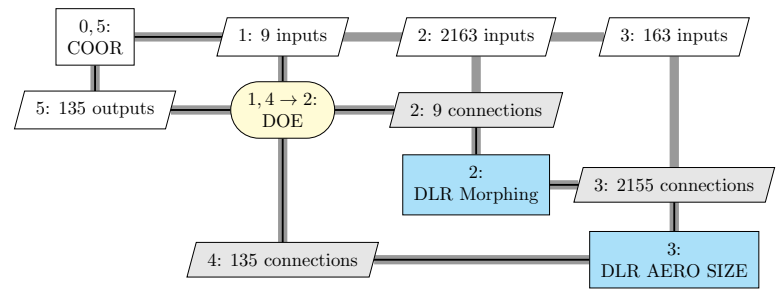

(c) DOE of Weight Cluster

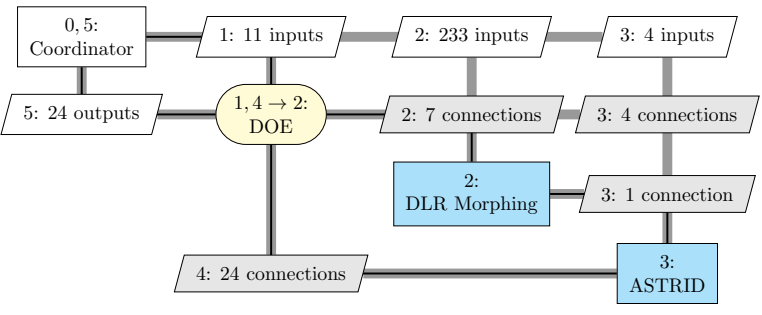

(b) DOE of System Cluster

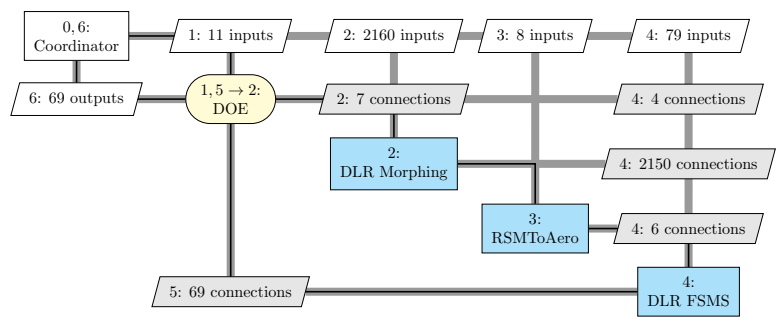

(d) DOE of Mission Cluster

Fig. 13 XDSM view of the DOE architectures for four competence clusters (two-step approach). The XDSMs are a visualization by VISTOMS package of the MDAO Data and Process Graph created by KADMOS.

- Step 1: A DOE sampling plan is generated by a specialized Partner, stored in a dedicated CPACS study branch, and provided to the Partner which is responsible for the integration and the execution of the DOE samples via the so-called DOE Study service.

- Step 2: The Partner responsible for the DOE Study service receives the complete sampling plan, and the contained input and output quantities are mapped to the parameters which need to be varied within the specific clusters' workflows. For the described DOE clusters, the sampling plan quantities have been mapped to the DLR aircraft geometry morphing tool, which provides the input (a CPACS aircraft) to the specific cluster's workflow to be executed for each DOE point. Note that such a cluster workflow is also hosted at a different Partner, which is responsible for the specific cluster's workflow integration, and offered as a remote service to the Partner initializing the DOE sampling plan in the previous step.

- Step 3: The specific cluster's workflow receives the DOE sample point as input, it is executed as a remote service requested in step 2. The specific cluster's workflow is also composed by multiple competences, which are CPACS compatible and hosted at different Partners' sites and provided as remote services. The list of the design competences used in the 4 clusters is provided in Section IV.A

The results obtained within the cluster's workflows (in step 3) are collected by the DOE Study service workflow (in step 2) and mapped back as DOE output for each sampling point, and the complete DOE database is provided back to the Partner initializing the DOE (in step 1). Afterwards, the DOE database stored as CPACS study branch is forwarded to the Partners responsible for the generation of the cluster surrogate models, or for the further enhancement of the DOE sampling plan.

For all the four DOE clusters shown in Figure 13, three steps (DOE generation, DOE study service and Specific Cluster workflow) have been implemented as individual RCE workflows hosted at different Partners' sites. The deployed approach makes use of the AGILE Collaborative Architecture's elements for requesting and providing the remote services. The complete process for the DOE sampling generation-execution-enhancement is fully automated. As described in Section IV.B each of the DOE has a specific set of input parameters, and output parameters provided by the distributed design competences which are selected for the cluster. Therefore, for each of the DOE 4 clusters illustrated by the XDSM in Figure 13, a selection of output parameters is shown in Figure 15, and briefly summarized in the following.

- Aero Cluster: wing planform parameters (aspect ratio and wing area displayed) are provided as DOE input to the cluster's workflow composed by the aerodynamics analysis modules provided by DLR and UNINA (maximum lift coefficient at take-off displayed). 


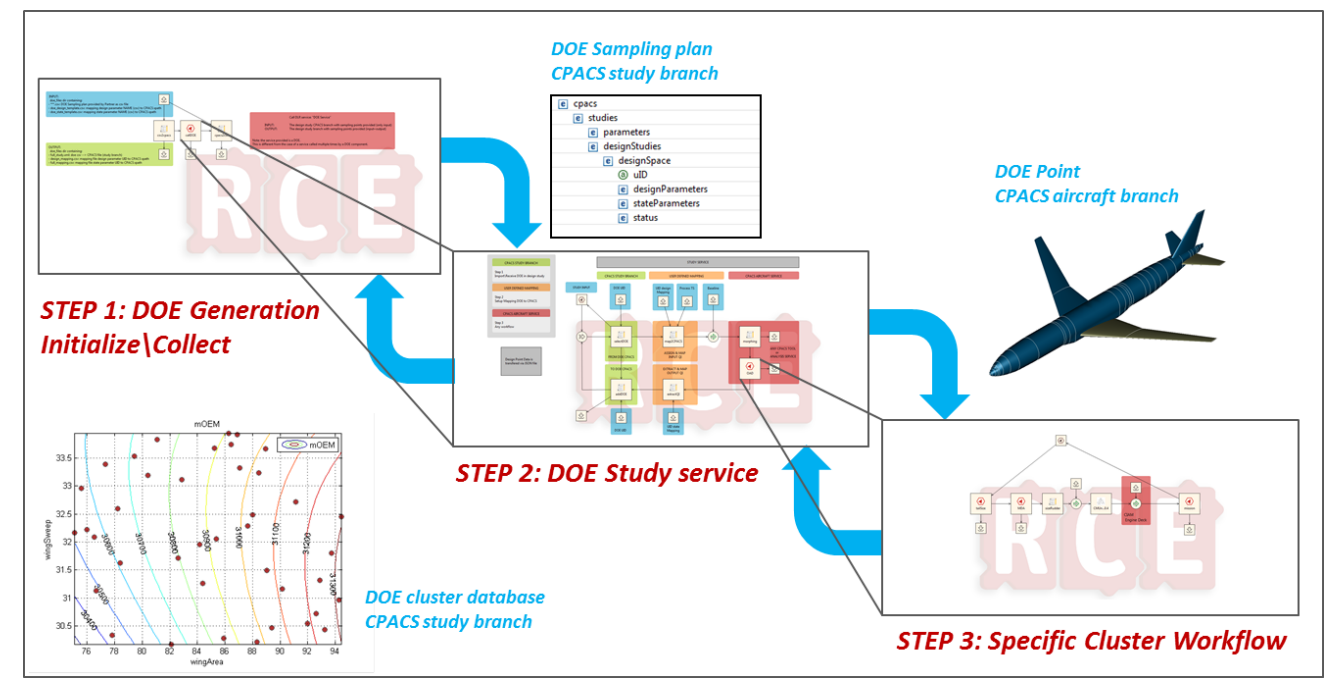

Fig. 14 Collaborative DOE study: nested steps approach

- System Cluster: wing planform (wing area displayed) and design masses (Maximum Take-Off Mass displayed) are provided as DOE input to the cluster's workflow composed by the on-board systems design competence provided by POLITO (mass of the sized on-board systems displayed).

- Weight Cluster: wing planform parameters (wing sweep angle and wing area displayed) are provided as DOE input to the cluster's workflow composed by the loads analysis and structural sizing design competence provided by DLR (operating Empty Mass displayed).

- Mission Cluster: wing planform (wing area displayed) and design masses (Operating Empty Mass displayed) are provided as DOE input to the cluster's workflow composed by the mission performance analysis module provided by DLR (mass of the mission fuel displayed).

\section{B. Surrogate models}

\section{Available methods}

Thanks to AGILE consortium, multiple methods are available regarding surrogate models (SM) competence. During DC-2, one of the objectives was to make these methods accessible to the partners through their integration as remote services in one of the PIDO framework, like any other design competences. Multiple SM competences were investigated in the frame of this use case.

- NLR's toolbox, called MultiFit [30] which provides a MATLAB based integration of multiple data fitting methods (e.g. polynomial, kriging, spline, neural network).

- ONERA's tool, MOE, a Mixture of Experts technique which combines local surrogate models [31]. The local expert could be a polynomial model (linear, quadratic, cubic), a radial basis function or some specific kriging models adapted to high-dimensional problems [32, 33].

- NOESIS Optimus kernel which provides a set of surrogate models and accuracy evaluation tools. Generated models can be evaluated from Optimus or exported and integrated in other application as executable or Functional Mockup Units.

- Surrogate model built by AIRINNOVA is Kriging \& co-Kriging [34] based on Matlab DACE toolbox [35].

\section{Modeling of the Aero Cluster}

This subsection provides an overview of the results obtained with MultiFit toolbox for the surrogate model (SM) of the Aerodynamic analysis cluster (AeroCluster). The AeroCluster takes as input the wing design parameters. As a result of the performed analysis the AeroCluster provides lookup tables for aerodynamic coefficients, related to the specified wing design. The lookup tables are given as function of the mission dependent parameters Mach, Reynolds Number (Re), Angle of Yaw (AoY) and Angle of Attack (AoA). The lookup tables will be used later on in the Mission 


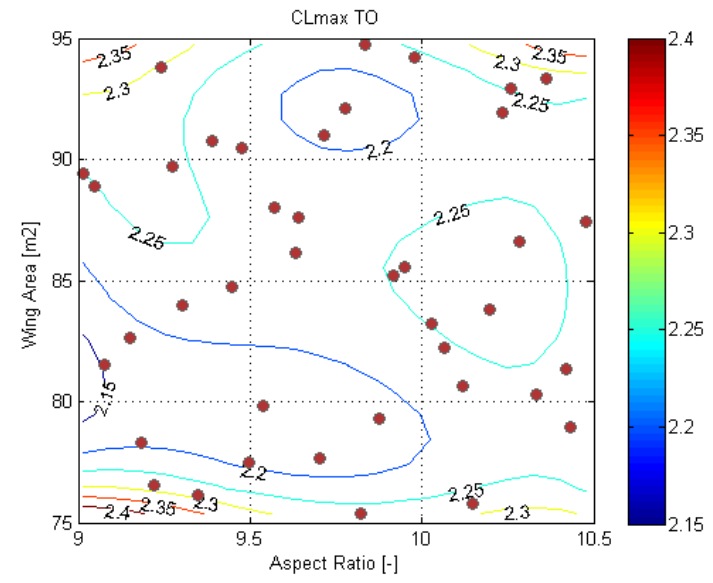

(a) DOE output of Aero Cluster: CLmax at take-off

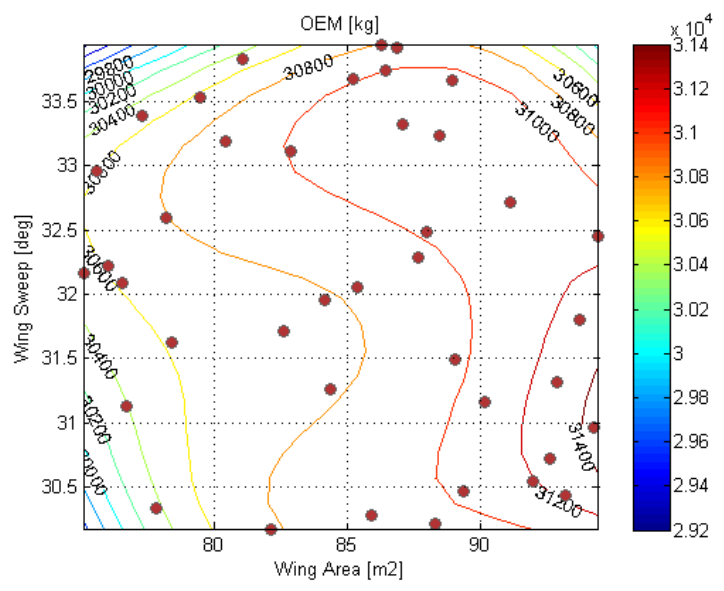

(c) DOE output of Weight Cluster: Operating Empty Mass (OEM)

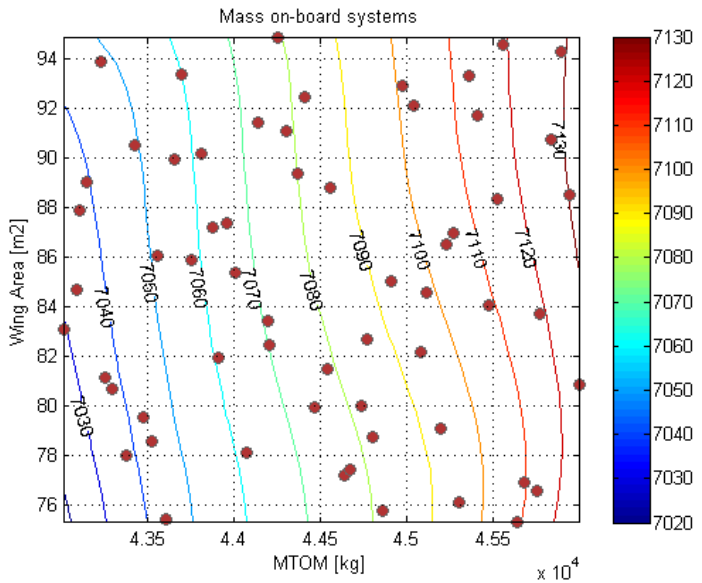

(b) DOE output of System Cluster: Mass on-board systems

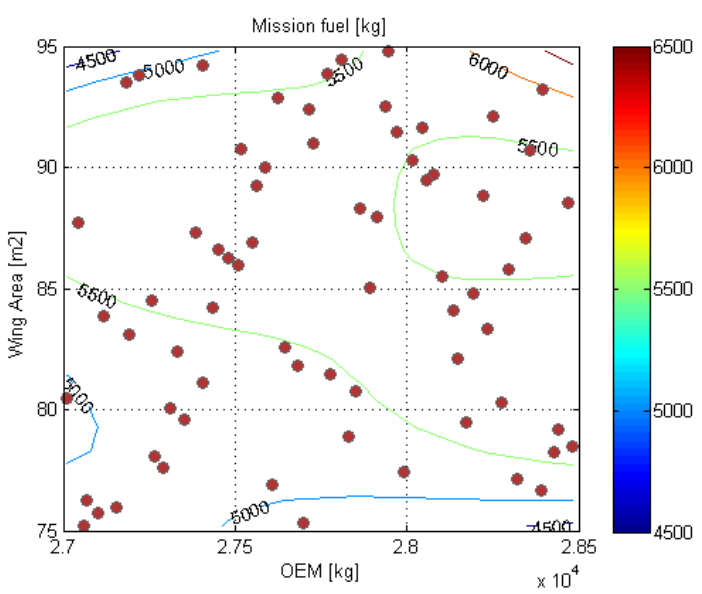

(d) DOE output of Mission Cluster: Mass mission fuel

Fig. 15 DOE clusters sample points results

performance Cluster, in order to calculate the actual values of the aerodynamic coefficients and from there contribute to the overall design objectives, e.g. fuel mass.

As already mentionned in Section IV.B two approaches have been identified in order to derive a surrogate model of the AeroCluster (AeroClusterSM) and relate it to the MissionCluster:

- A two-step approach. In this case the AeroClusterSM predicts - as a function of wing design parameters - a (representation of) the aero lookup tables, e.g. by predicting polynomial coefficients. This approach is illustrated in Figure 16 .

- A "all-in-one" (AI1) approach. In this case the AeroClusterSM directly predicts the aerodynamic coefficients as a function of wing design parameters and mission parameters. As such the AeroClusterSM becomes an integrated part of the MissionClusterSM. This approach is depicted in Figure 17

As indicated in Section V.A, a database of 40 aircraft configurations (with varying wing design parameter values) have been processed by the AeroCluster. The aero table parameter AoY was not varied and is therefore ignored. Below surrogate model derivations are described both for the two-step and the AI1 approaches. In each case the surrogate model is created using a training set based on the first 39 configurations. The $40^{\text {th }}$ configuration is used for validation of the surrogate models. Only the first aerodynamic output Cfx is considered here, for simplification. The other aerodynamic coefficients (e.g. Cfy, Cfz) could be predicted in a similar fashion. Both two-step and AI1 approaches have been investigated.

Two-step approach With this approach two methods for creating the AeroCluster SM have been applied. 


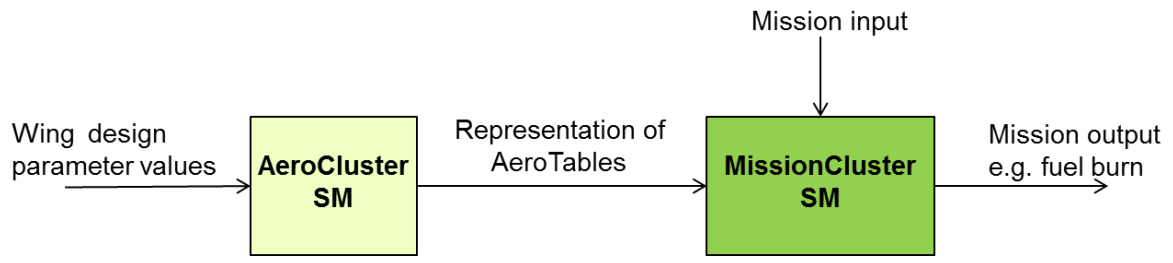

Fig. 16 Depiction of the "two-step" approach. For simplicity the other clusters have been left out here.

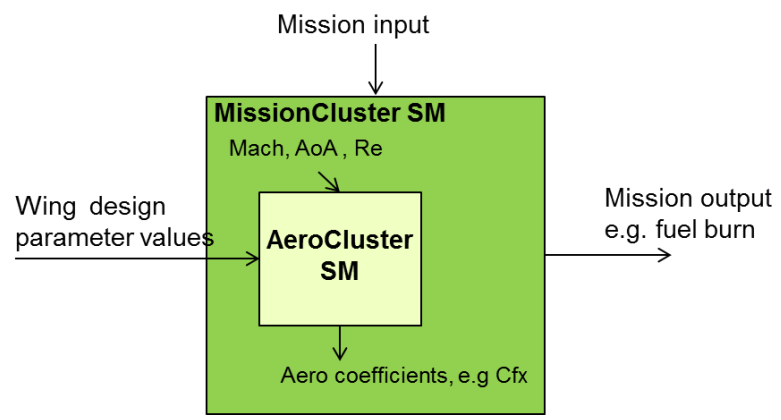

Fig. 17 Depiction of the "all-in-one" (AI1) approach. For simplicity the other clusters have been left out here.

- Method1: Approximation of the aero lookup tables using polynomials and prediction of these polynomial coefficients (as function of wing design) again using polynomials.

- Method2: Prediction of the aero lookup tables using Proper Orthogonal Decomposition (POD) [36] and polynomial prediction of the POD coefficients (as function of wing design).

1) Method1: Figure 18 shows the result of stepwise polynomial fits of the lookup tables of all 40 configurations. For all configurations the stepwise fit resulted in a $2^{\text {nd }}$ order polynomial (as a function of Mach, Re and AoA) with 7 coefficients: using the constant term, three linear terms, the cross term Mach $\times$ AoA and the quadratic terms $\mathrm{Mach}^{2}$ and $\mathrm{AoA}^{2}$. Figure 18 shows that all 40 sets of polynomial coefficients have a piecewise similar order of magnitude. Therefore they could be fitted as well as function of the design configuration. The sets of polynomial coefficients have again been stepwise fitted by a 2 nd order polynomial, leaving the $40^{\text {th }}$ polynomial out for validation. This last stepwise fit represents the AeroCluster SM. The $40^{\text {th }}$ polynomial (consisting of 7 coefficients) has been predicted with the AeroCluster SM and has been applied in order to predict the aero lookup table values of the $40^{\text {th }}$ configuration. The results are depicted in Figure 19. A maximum absolute Cfx prediction error of less than 0.02 has been achieved. Figure 19 shows that this corresponds to a relative error of about 10 percent. Other methods (e.g. kriging) for fitting the set of polynomial coefficients have been tried as well, but they gave worse prediction results on the $40^{\text {th }}$ configuration than the stepwise fit AeroCluster SM. Concluding, the AeroCluster SM derived with this method predicts 7 polynomial coefficients that can be passed on to the Mission Cluster for prediction of the aerodynamic coefficients later on.

2) Method2: When the lookup table values of Cfx are ordered into one long row (of 192 samples) they can be considered as a "snapshot" of the $i^{\text {th }}$ aircraft configuration. This results in a "snapshot-matrix" $A$ of $40 \mathrm{x}$ 192. A can be reduced using POD. A singular value decomposition

$$
A=U \times S \times V^{T}
$$

is derived. From $S$ the dominant singular values are selected, in this case the first three. The matrix $U \times S$ contains the POD vectors (row-wise). The first three columns of this matrix are to be fitted, as a function of aircraft configuration. The $40^{\text {th }}$ row of $U \times S$ is left out and the remaining 39 rows are fitted, again using stepwise fit of a 2 nd order polynomial. The $40^{\text {th }} \mathrm{POD}$ vector is predicted using this polynomial and back transformed to a prediction of the $40^{\text {th }}$ row of $A$ ( $40^{\text {th }}$ aero table "snapshot"). The results are depicted in Figure 20 This result is a maximum prediction error of less than 0.01 , which is better than the validation result with Method1. 


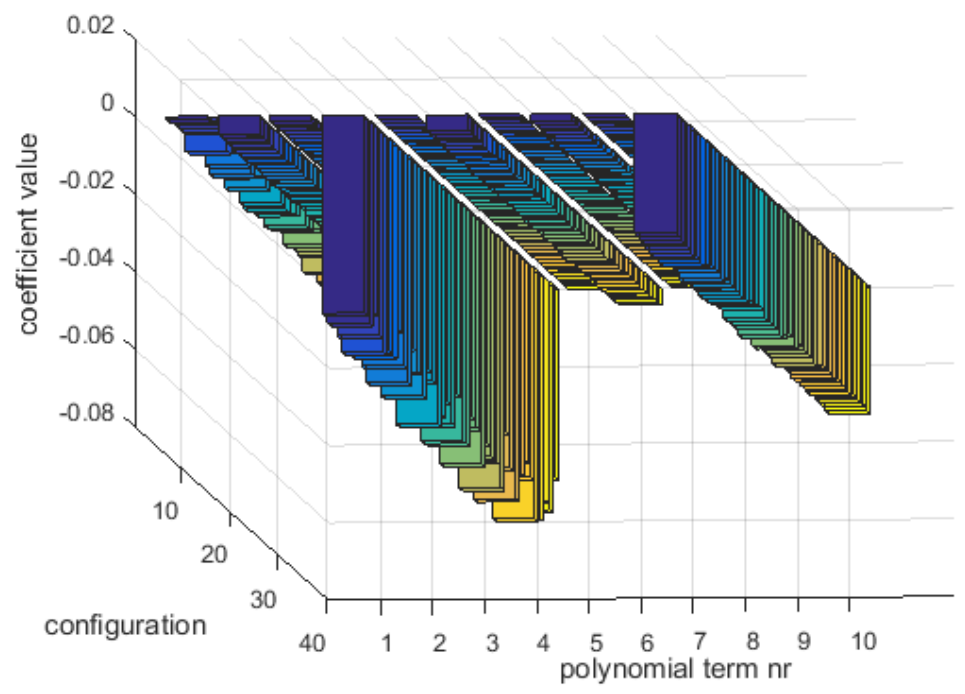

Fig. 18 Coefficient values of a 2nd degree polynomial (derived with stepwise fit of the aero lookup tables), for all 40 aircraft configurations

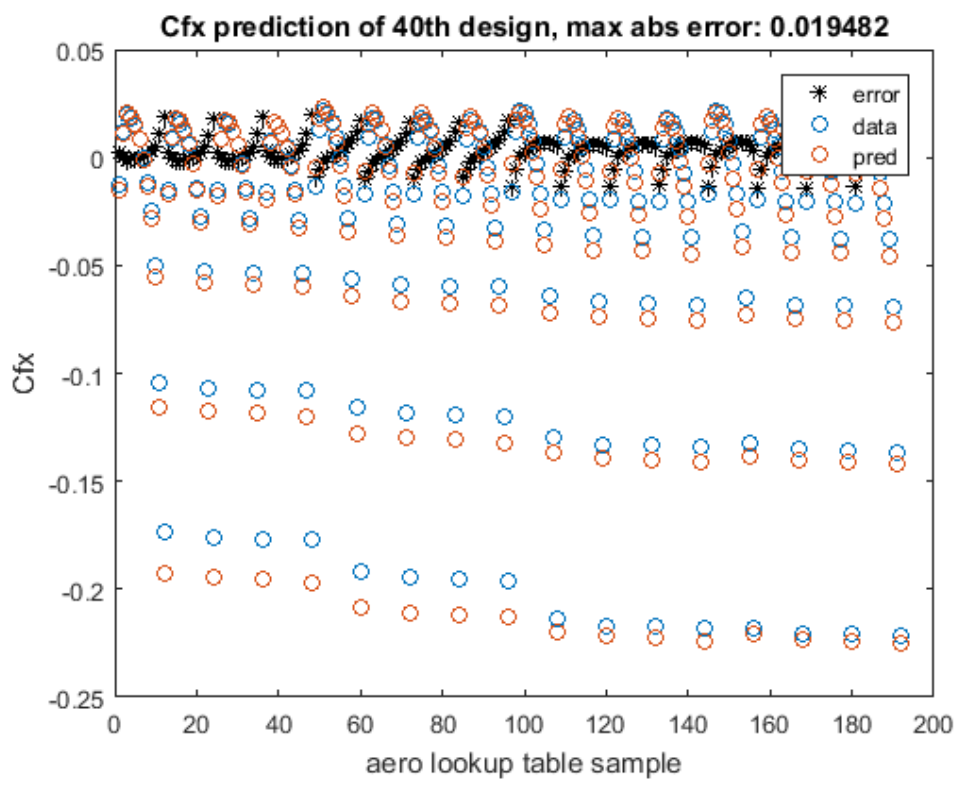

Fig. 19 Prediction of the $\mathrm{Cfx}$ aero table values on the $40^{\text {th }}$ configuration : Method1 with Nested stepwise polynomial fits

Concluding, the AeroCluster SM derived with this method is composed by a stepwise polynomial fit and POD transformation functions. The AeroCluster SM predicts the full aero lookup table (as function of wing design parameters) which can be passed on to the mission cluster for prediction of the aerodynamic coefficients later on.

All-in-one approach With the AI1 approach the 7 wing design parameters and the 3 mission parameters have been combined, resulting in a dataset of $40 \times 192=7680$ points, with 10 inputs and 1 output (Cfx). An Artificial Neural Network (ANN) has been fitted on to the first 7488 points, which correspond with the 39 configurations. One 


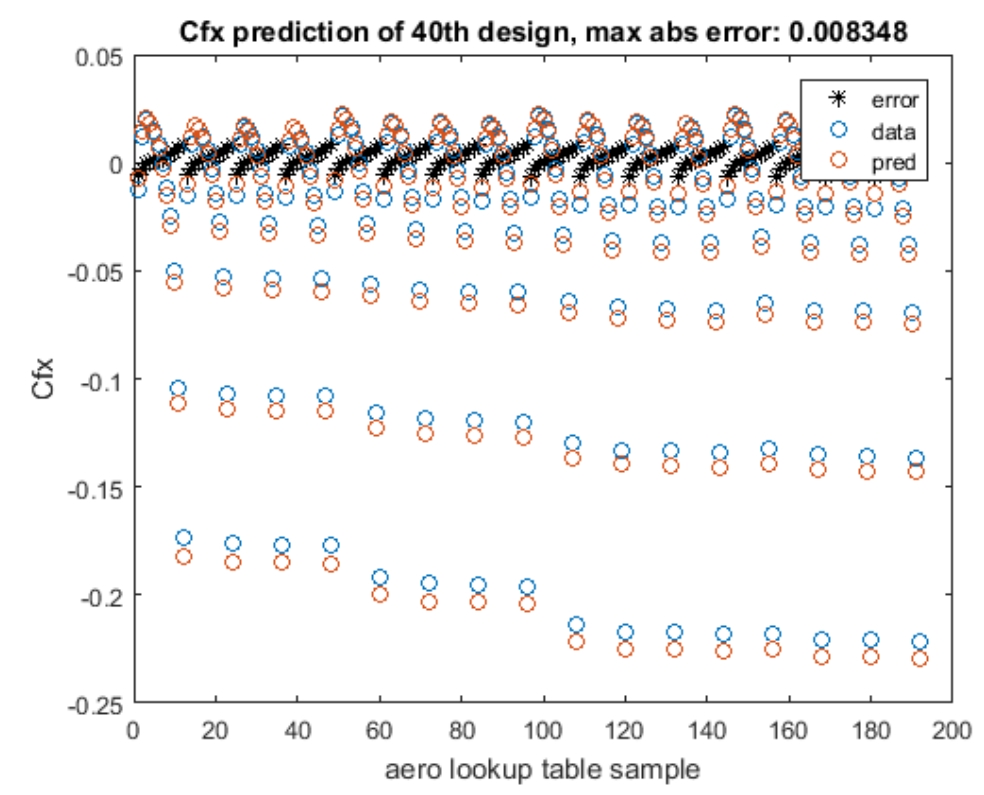

Fig. 20 Prediction of the $\mathrm{Cfx}$ aero table values on the $40^{\text {th }}$ configuration using Method2 (stepwise polynomial fit of POD vectors)

hidden layer with 10 neurons has been used. The aero lookup table values of the $40^{\text {th }}$ configuration are predicted as validation of the method. The results are depicted in Figure 21. This result is a maximum prediction error of less than 0.01 which is comparable to the validation result with Method 2 of the previous approach.

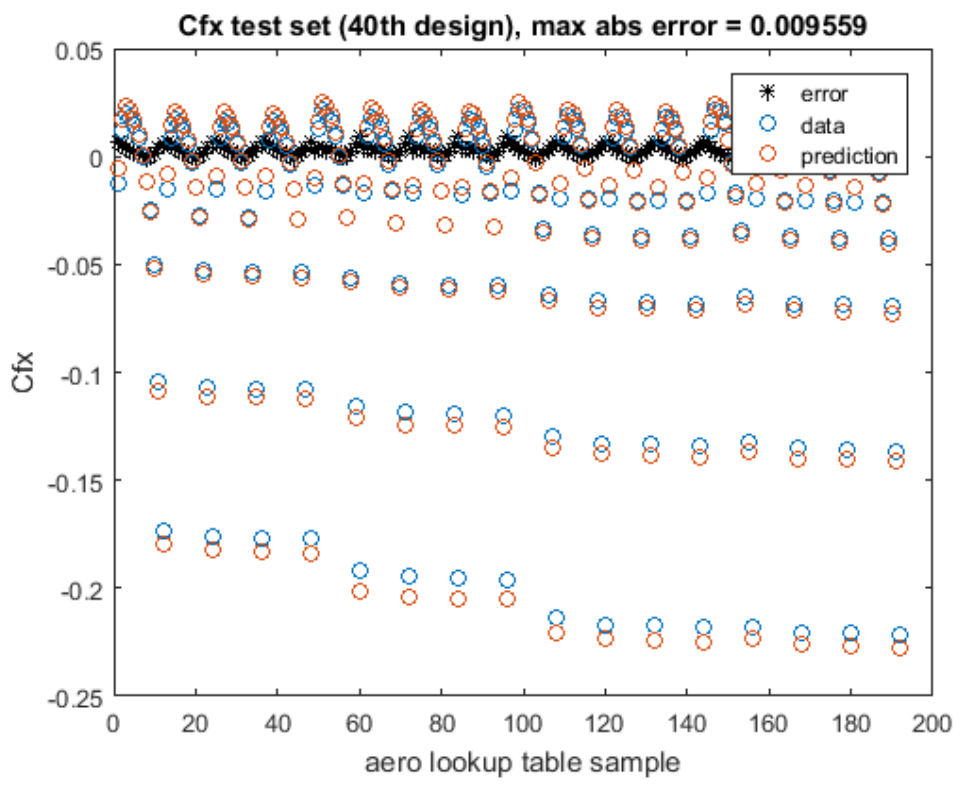

Fig. 21 ANN prediction of the CfX aero table values on the $40^{\text {th }}$ configuration based on the AI1 approach

Concluding, the AeroCluster SM derived with this approach consists of an ANN that must be integrated with the

MissionCluster SM, as illustrated in Figure 17

Similar investigations were performed by other partners on the AeroCluster and similar approach was also applied to the other clusters once the database was made available. One should note that the objective, here, was not to benchmark the various SMs of the partners but to make accessible multiple surrogate Design Competences through the AGILE 
framework to build the surrogate-based MDA workflow. In the next chapters, the "all-in-one" approach has been retained.

\section{MDA clusters workflow}

This paragraph presents the next step of the approach: the building of the MDA workflow of surrogate models in a PIDO framework. The results shown here were obtained using the Optimus framework [23] and the surrogate models were provided by NOESIS

To illustrate the approach, the four clusters have been embedded in a single Optimus MDA workflow depicted on Figure 22 that reproduces the simplified connection schema depicted in the XDSM (see Figure 10). As the SMs were already available on the same platform, a non-collaborative implementation (ie. not using Brics) of the MDF architecture has been preferred. This direct implementation has been made possible by the clusters created using the knowledge-based part of the AGILE framework, that minimized the number of variables and connections from the complexity represented in Figure 10 to something manageable by hand. The information exchange between the clusters has been managed using the Optimus native variables. The SMs, exported as binary models, have been prepared for execution via an external evaluator (thus their analysis is triggered from within the workflow, but run independently). The highlighted blocks in the Figure 22 are:

\section{Yellow color: Aero Cluster Green color: Structural sizing and Weight Cluster \\ Red color: On-board systems Cluster Azure color: Mission Cluster}

Thanks to AGILE developments, the structure of the workflow can be adapted to a collaborative workflow with minimal efforts following the collaborative framework methodology; each colored block can be replaced with a 3-components assembly that performs the CPACS mapping, Brics task creation and extraction of the output values from the enriched CPACS. The operations with the surrogate models operated as remote discipline have been successfully tested using the multi-task feature of Brics.

In this implementation, there are two nested convergence loops (represented by the circular icons on Figure 22). They are required as the connections among the tools, which have been severed for the DOEs, have to be re-established to ensure the MDA functionality; in some cases this implies that a cluster may receive the input before the corresponding output has been generated by another cluster. To this end, a nominal initialization value, subsequently refined in the following iterations, has been used. Each loop has been addressed using a fixed point iteration until the assigned tolerance is reached (absolute relative difference between two successive iterations lesser than $0.1 \%$ ). The inner loop connects weight and system clusters and is required to ensure the consistency of the systems weight value (mSystem coupling variable). The outer also includes the Mission cluster and is mandatory to achieve convergence on the fuel weight (mFuel coupling variable) information. On average to achieve convergence on both loops, 45 evaluations have to be performed ( 9 runs of the outer loop, each requiring 5 runs of the inner). Input values have been gathered on the top of the workflow, corresponding to WingGeometry parameters. The design variables for Structural sizing and Weight and On-board systems Clusters are either inherited or generated as output. System and fuel mass are not to be considered independent design variables.

A single run of the non-collaborative MDA takes about 30 seconds; on average (could vary due to specific design variable values) 5 iterations of the internal loop and 9 of the outer one are required to achieve the convergence requirements. The total number of calls to the SM evaluator in an average case is 100 as reported in Table 2 . The equivalent collaborative workflow (ie. using Brics) requires around 300 seconds, due to the overhead introduced by data upload and download on the sharedpoint server. The overhead is particularly relevant using SM because of the significantly reduced execution time of the modeled discipline, from seconds/minutes to less than a second. Apart from the different execution time, both collaborative and non-collaborative implementations have been validated and produce equivalent simulation results. The non-collaborative version of the MDA has been used for the following analysis.

Table 2 SM evaluator calls for each discipline for a nominal run

\begin{tabular}{c|c|c|c|c} 
& Aero cluster SM & Weight cluster SM & Systems cluster SM & Mission cluster SM \\
\hline Number of Calls & 1 & 45 & 45 & 9
\end{tabular}

Thanks to the reduced execution time it has been possible to perform a DOE to explore the design space and investigate the impact of the design variables. A 100 experiments Latin Hypercube Sampling has been used. The 


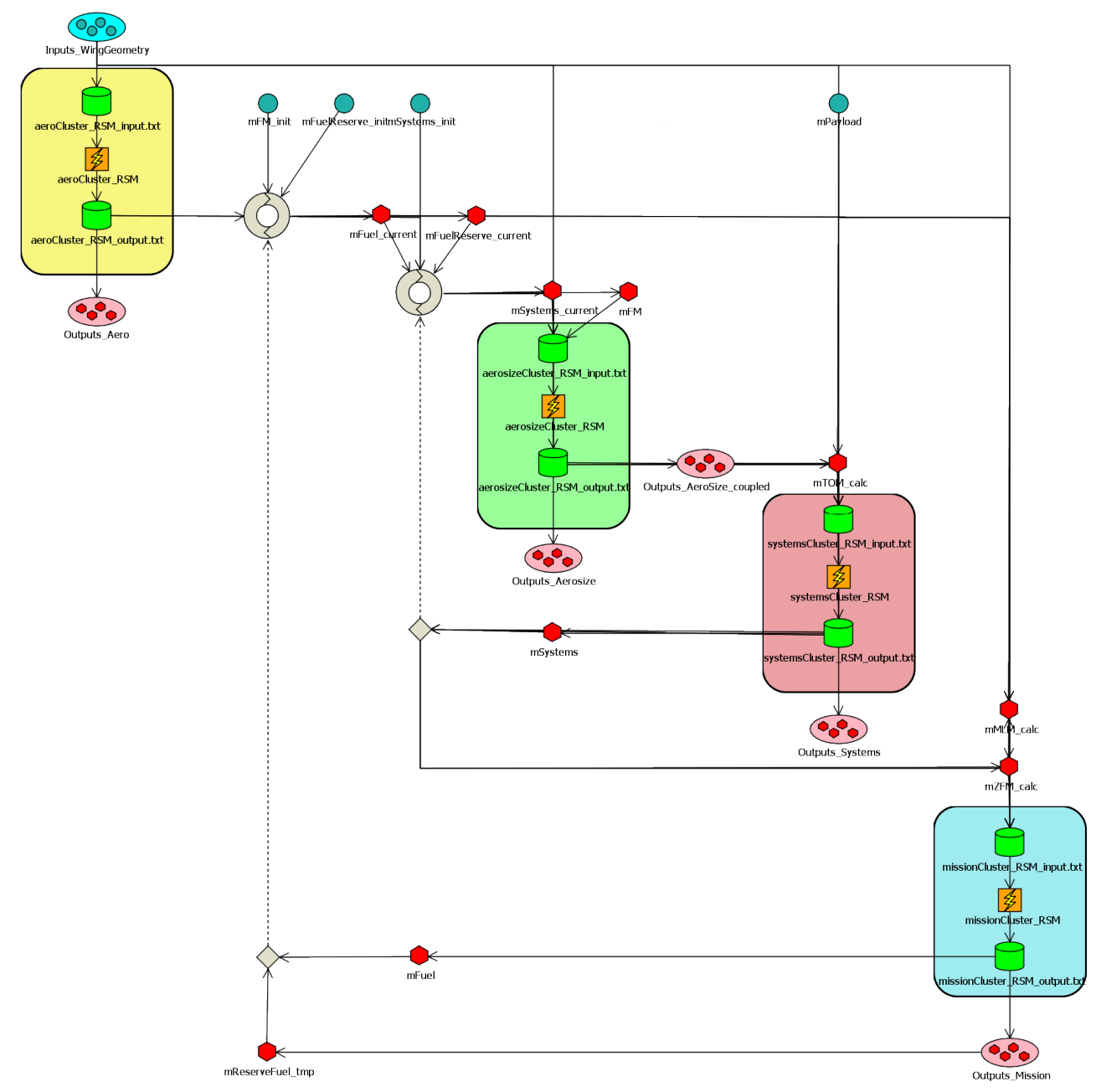

Fig. 22 MDA through surrogates with four clusters

analysis of the Pearson (measure of the linear correlation between two variables) coefficients has been reported in Figure 23. Values can vary between +1 (total positive linear correlation) and -1 (total negative linear correlation). Colors are proportional to the absolute value of the coefficient and emphasize outputs that are significantly affected by a specific design variable (i.e. wing mass and wing area). Small correlation values between inputs are due to the selected DOE algorithm, the Latin Hypercube, that does not ensure complete orthogonality in the design space exploration. As an example in Figure 23, are highlighted the (almost linear) influence that the wing area (wingArea) has on Wing Total Mass (mWing) and Operative Empty Weight (mOEM) and the negative effect on both the evaluated lift coefficients (CLmaxTO and CLmaxL) due to wing tip twist (twistTip).

The MDA has been used to perform a first optimization aimed at minimize the maximum Take Off Mass with a constraint on the minimum range $(3450 \mathrm{~km})$. The optimization problem can be defined as follows:

$$
\begin{cases}\text { min } & \text { maximum Take Off Mass: } \text { mTOM } \\ \text { w. r. t. } & 7 \text { wing shape variables } \\ \text { s. t. } & 3450000-\text { range }<0 .\end{cases}
$$

A global search method, the Self Adaptive Evolution [23] has been selected as optimization algorithm. The method performs an expensive initial exploration of the entire design space but is robust against local minima. The evolution of design variables, outputs and objective has been reported in Figure 24. The colors represent the iteration number; a convergence pattern is clearly visible. The final configuration has been reported in Table 3

The optimization algorithm selected a thinner and narrower wing in order to minimize drag and consequently fuel 


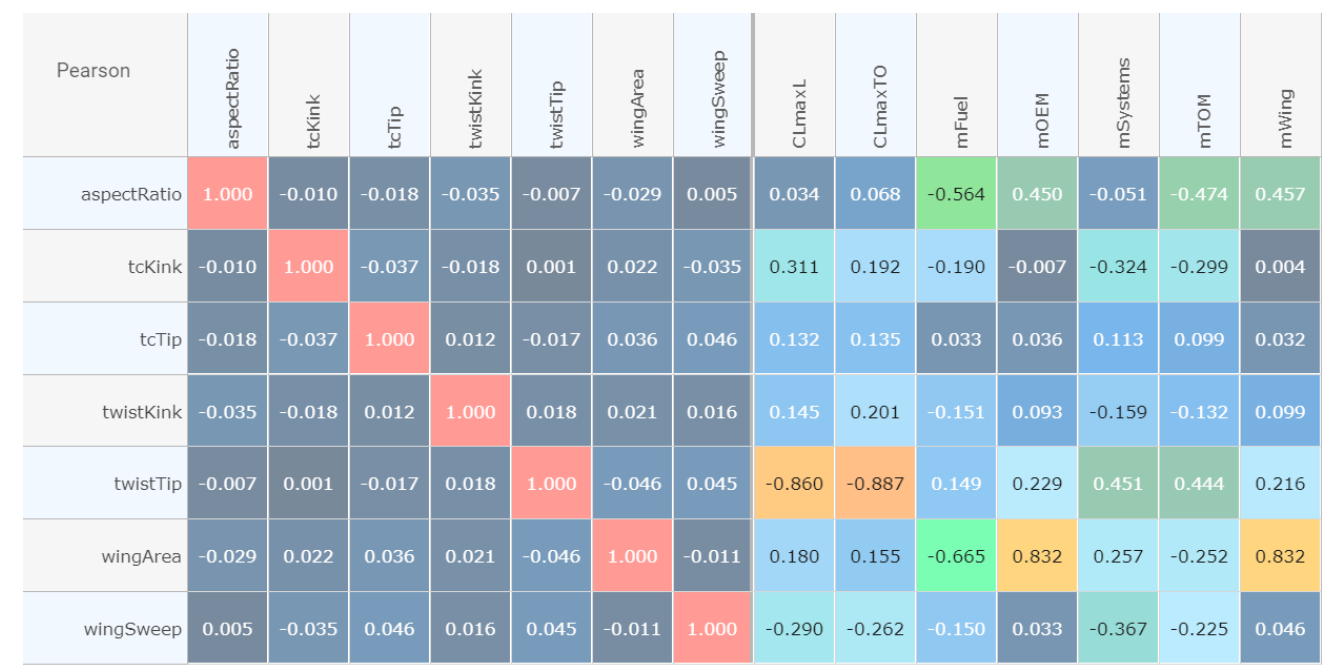

Fig. 23 MDA through surrogates, design variable influence on evaluated outputs - Pearson coefficient

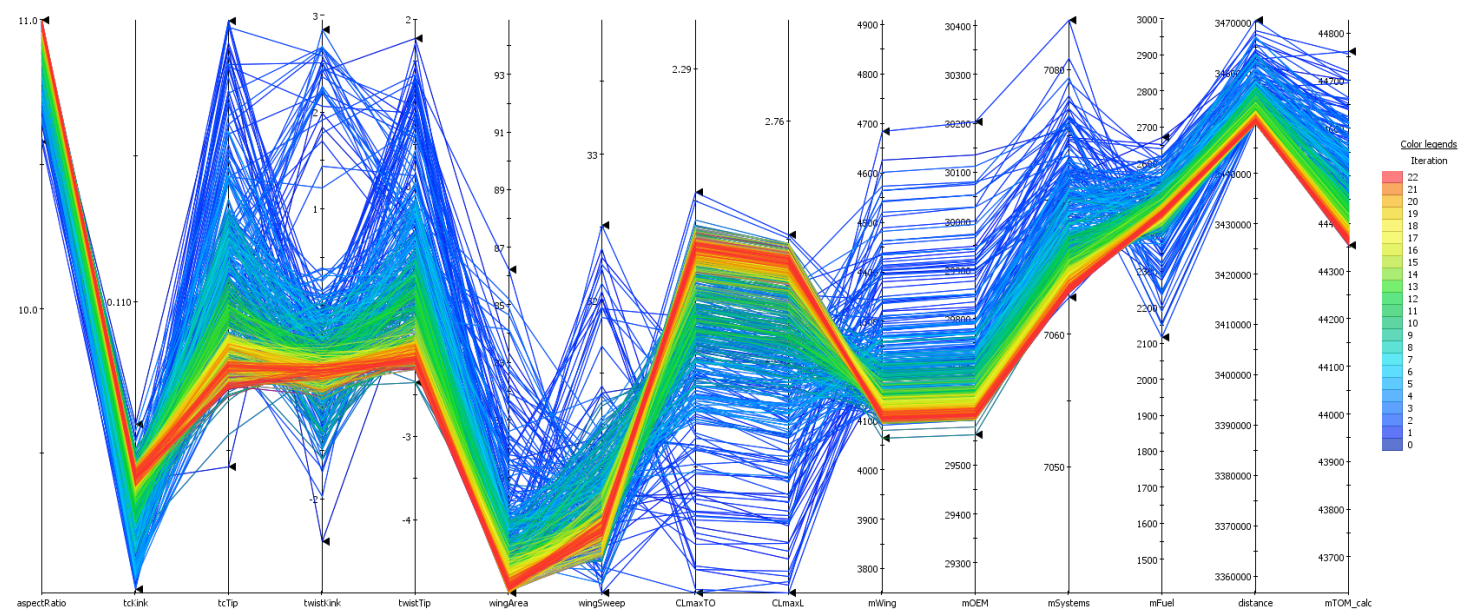

Fig. 24 MDA through surrogates, parallel coordinates for constrained mTOM minimization

consumption; the range constraint achievement has been accompanied by an estimated reduction of total fuel mass. The optimization has been performed only to test the MDA through surrogates functionality and the achieved results are for demonstration purposes only.

\section{MDO formulations}

As in previous section, both target MDA and a first optimization were successfully evaluated in Optimus, the last step of the overall process will be to test the scenario related to the investigations of MDO formulations described in Section III (scenario 1). This activity was made possible by the use of MDO support applications such as VISTOMS, KADMOS and CMDOWS, which ease the setup and operation of classical MDO formulations (MDF and IDF) for complex workflows. An unconstrained mOEM minimization problem was selected:

$$
\begin{cases}\min & \text { Operating Empty Mass: } m O E M \\ \text { w. r. t. } & 7 \text { wing shape variables }\end{cases}
$$

The range of design variables are similar to values given in Table 3. For the IDF formulation, that requires coupling variables as design variables, a range had to be defined in line with the one used in the cluster DOEs. Starting from 
Table 3 mTOM minimization, initial (unfeasible) and final configurations

\begin{tabular}{|c|c|c|c|c|}
\hline & Start & End & Lower bound & Upper bound \\
\hline Inputs & \multirow[b]{2}{*}{10.} & \multirow[b]{2}{*}{11.0} & \multirow[b]{2}{*}{9.} & \multirow[b]{2}{*}{11.} \\
\hline aspectRatio & & & & \\
\hline tcKink & 0.11 & 0.105 & 0.1 & 0.12 \\
\hline tcTip & 0.1 & 0.11 & 0.09 & 0.11 \\
\hline twistKink & 0. & 2.99 & -3 & 3. \\
\hline twistTip & -1.5 & -4.98 & -5 & 2. \\
\hline wingArea $\left[\mathrm{m}^{2}\right]$ & 85. & 76.43 & 75. & 95. \\
\hline wingSweep & 32. & 30.01 & 30. & 34. \\
\hline Outputs & \multirow[b]{2}{*}{2.2585} & \multirow[b]{2}{*}{2.376} & & \\
\hline CLmaxTO & & & & \\
\hline CLmaxL & 2.7255 & 2.855 & & \\
\hline mWing $[k g]$ & 4330.99 & 4105.49 & & \\
\hline mOEM $[k g]$ & 29863.54 & 29602.03 & & \\
\hline mSystems $[k g]$ & 7097.68 & 7061.78 & & \\
\hline mFuel $[k g]$ & 3129.83 & 2454.32 & & \\
\hline range $[m]$ & 3446740 . & 3450336 . & & \\
\hline $\operatorname{mTOM}[k g]$ & 44281 & 44388.23 & & \\
\hline
\end{tabular}

the CPACS[37] I/O of each cluster, the building of the MDO formulations such as MDF or IDF were set-up using VISTOMS[27] with KADMOS[26]. Eventually, CMDOWS[28] files of the MDO solution strategy (see Figure 8) were automatically created. These CMDOWS files contain a neutral description of the workflow that should be executed and can be opened in any PIDO framework used in AGILE, such as RCE or Optimus. Figure25 provides the VISTOMS XDSM, the RCE and the Optimus views of both MDO formulations (MDF and IDF) for the retained MDO problem (2).

Using these CMDOWS files, optimizations were run both in Optimus and RCE to evaluate some mono-objective optimization algorithms. In RCE, both COBYLA [38], NOMAD[39], from DAKOTA package [40], were applied. In NOESIS Optimus framework, EGO [41] and standard Particle Swarm Optimization (sPSO) algorithms [42] were tested. In addition, ONERA's optimization algorithm, SEGOMOE [7, 43, 44], used in DC-1 (see Section [II), and relying on ONERA's MOE surrogate models III was also benchmarked. Table 4 presents some examples of the results obtained for MDF and IDF formulations.

Table 4 Preliminary results for the MDA through surrogate test case obtained using various optimization algorithms, MDF or IDF formulation and RCE or Optimus framework to solve problem (2).

\begin{tabular}{|c|c|c|c|c|c|c|}
\hline \multirow{3}{*}{$\begin{array}{l}\text { PIDO } \\
\text { MDO Formulation } \\
\text { Optimizer }\end{array}$} & \multicolumn{4}{|c|}{ RCE } & \multirow{2}{*}{\multicolumn{2}{|c|}{$\begin{array}{c}\text { Optimus } \\
\text { MDF }\end{array}$}} \\
\hline & \multicolumn{3}{|c|}{ MDF } & \multirow{2}{*}{$\begin{array}{c}\text { IDF } \\
\text { NOMAD }\end{array}$} & & \\
\hline & COBYLA & NOMAD & SEGOMOE & & EGO & sPSO \\
\hline \multicolumn{7}{|l|}{ Inputs } \\
\hline aspectRatio & 9. & 9. & 9. & 9.005 & 9. & 9. \\
\hline tcKink & 0.12 & 0.12 & 0.12 & 0.12 & 0.1127 & 0.11 \\
\hline tcTip & 0.09 & 0.09 & 0.09 & 0.09 & 0.09 & 0.09 \\
\hline twistKink & -3 & -3 & -3 & -2.98 & -3 & -3 \\
\hline twistTip & -5 . & -5 & -5 & -5 & -4.96 & -5 \\
\hline wingArea $\left[\mathrm{m}^{2}\right]$ & 75. & 75. & 75. & 75. & 75. & 75. \\
\hline wingSweep & 30. & 30. & 30. & 30. & 30. & 30. \\
\hline \multicolumn{7}{|l|}{ Outputs } \\
\hline mOEM $[k g]$ & 29138.52 & 29138.52 & 29138.52 & 28706.46 & 28934.75 & 28932.81 \\
\hline
\end{tabular}




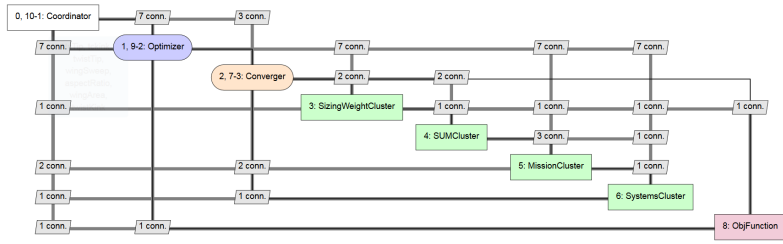

(a) XDSM of MDF

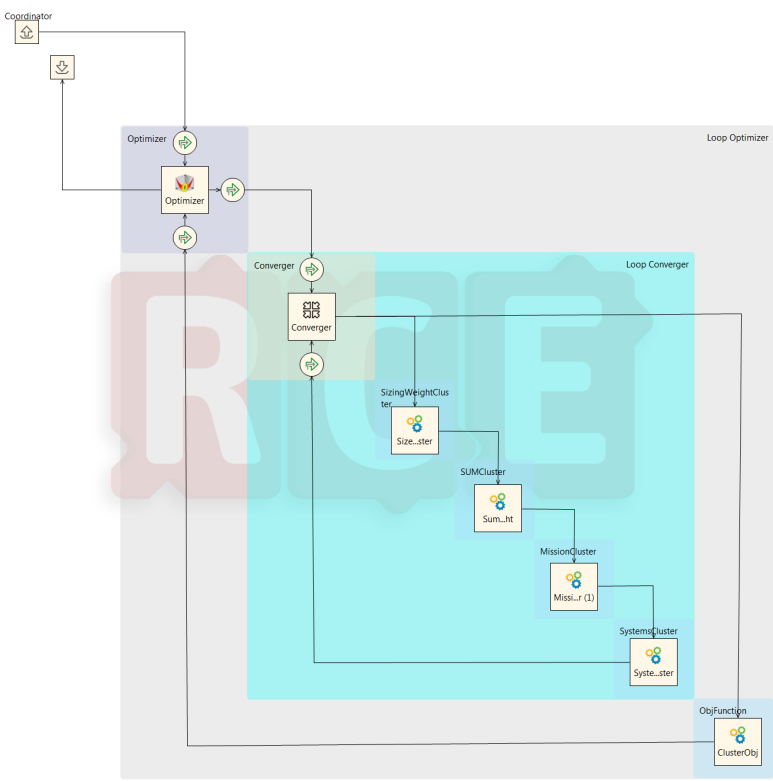

(c) MDF in RCE

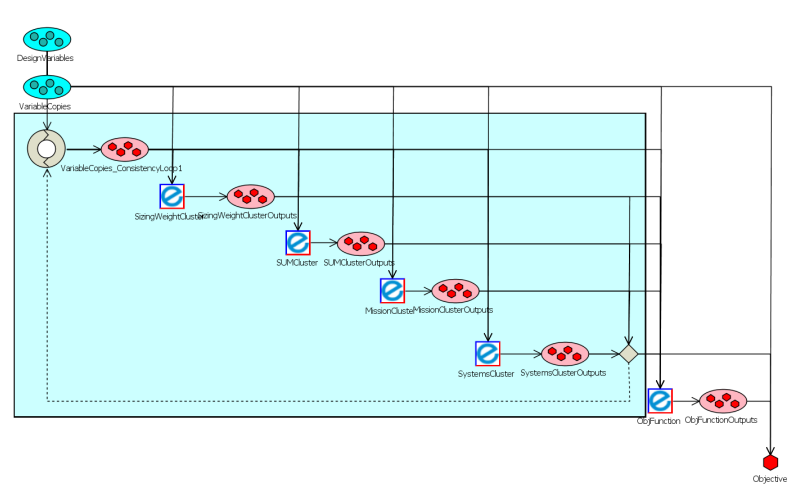

(e) MDF in Optimus

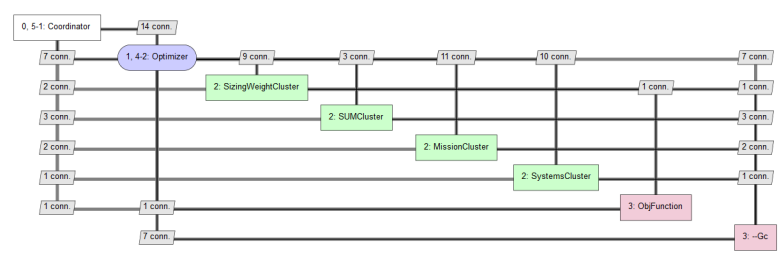

(b) XDSM of IDF

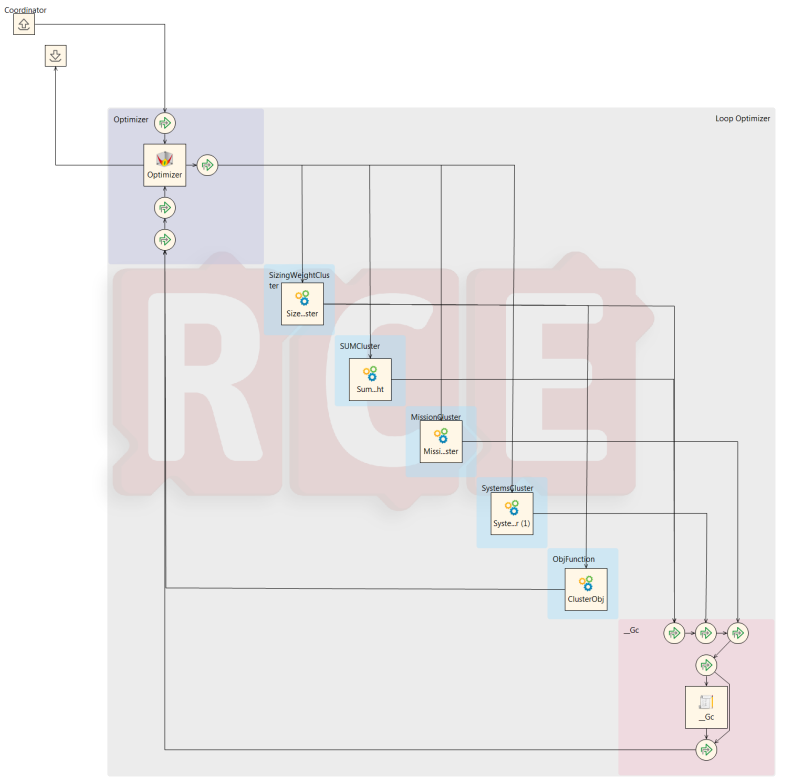

(d) IDF in RCE

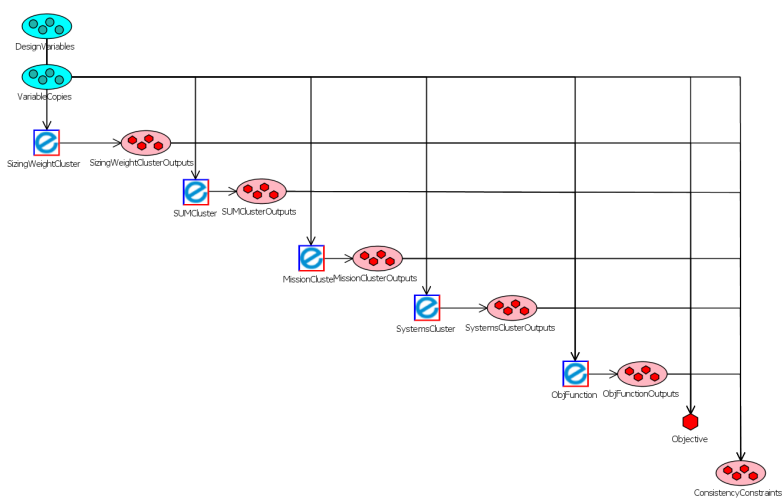

(f) IDF in Optimus

Fig. 25 VISTOMS XDSM, RCE and Optimus views of the MDF and IDF formulations.

One can check that all the optimizations converge towards a similar minimum even though they exhibit some slight differences for some design variables and objective function. Concerning the PIDO execution, the difference between RCE and Optimus results is mainly explained by the optimizer and surrogate models used. In addition, the slight discrepancy observed for the optimal design variable tcKink can be explained by the almost negligible effect of tcKink on mEOM highlighted in Figure 23. Concerning differences between IDF and MDF, in RCE platform (using the same surrogate models), the small gaps come from the fact that consistency constraints introduced by the IDF formulation were relaxed to a threshold of $10^{-2}$ to ease the convergence. 
Nevertheless, as targeted in scenario 1 (defined in Section III.A), a reference workflow has been made available to benchmark various MDO formulations and/or several kind of optimizers). Even though this workflow is already suitable, ways of improvement can be obtained with the enrichment of the databases or the increase of its complexity, like adding local variables in some clusters to enable the benchmark of multi-level MDO formulations.

\section{Conclusion and perspectives}

An innovative approach has been presented in this paper, in which knowledge-based engineering and collaborative engineering techniques have been used to handle a complex aircraft design workflow and apply it for an efficient MDO. The design workflow has been successfully simplified and implemented using surrogate models for clusters of disciplines to reduce the computational time. The activities are part of the EU H2020 project AGILE. Taking advantage of the improvements brought by the AGILE knowledge-based architecture, the setup phase of the process was strongly eased and the clustering of design competences was investigated in a collaborative way involving all tool specialists. An opportunity for improvement here concerns the clustering process, which could be partially automated as it currently still is an expert-based and manual process.

During the operational phase, the features implemented in the collaborative architectures, such as DOE service workflow, enabled a quick execution of all the DOEs for all the clusters, each one being a distributed workflow of partners' design competences. In addition, multiple methods regarding the creation of surrogate models (SM) were investigated and the SM were made available to the partners in different formats, such as directly executable SM or SM availability by remote access through a PIDO framework. Eventually, the target workflow, coupling the surrogate models of the clusters, was successfully implemented in the PIDO tool Optimus and provided encouraging preliminary results, thus demonstrating the success of the proposed approach.

In addition, this use case has also been used as a mock-up for testing the automation of the execution phase of an MDO system, enabled by KADMOS through the CMDOWS format, and enabled the application of classical and innovative MDO formulations. The next step will be to evaluate the optimal configuration with the real MDA in order to quantify the error induced by the simplified process and the MDA workflow and to carry on the investigation of adapted MDO formulations, potentially with an increase of complexity (adding local variables in some clusters for instance). Using the current workflow, preliminary results have already been obtained for innovative MDO formulation, called EGMDO, considering the propagation of modeling uncertainties induced by the errors associated to the use of surrogate models in the MDA resolution[21]. Last but not least, the use of surrogate models for clusters of design competences has proven to be a feasible and effective approach and is currently applied to novel configurations, such as strut-braced wing aircraft, in context of the final design campaign of AGILE.

Within the MDO community, many challenges have been identified [6, 25, 45] to ease the optimization of complex workflows in the context of multilevel and multipartner collaborative engineering projects that characterize aircraft design. However, it can be concluded that the technical solutions developed in AGILE, by smart combination of knowledge-based technologies, IT solutions and MDO strategies, provide a fruitful approach for handling those challenges and therefore contribute to a shorter aircraft development time.

\section{Acknowledgments}

The research presented in this paper has been performed in the framework of the AGILE project (Aircraft 3rd Generation MDO for Innovative Collaboration of Heterogeneous Teams of Experts) and has received funding from the European Union Horizon 2020 Programme (H2020-MG-2014-2015) under grant agreement $n^{\circ}$ 636202. The authors are grateful to the partners of the AGILE consortium for their contribution and feedback.

\section{Open-source references}

$\begin{array}{ll}\text { CMDOWS repository } & \text { http://cmdows-repo.agile-project.eu } \\ \text { CMDOWS interface } & \text { http://cmdows.agile-project.eu } \\ \text { CPACS repository } & \text { https://github.com/DLR-LY/CPACS } \\ \text { KADMOS repository } & \text { https://bitbucket.org/imcovangent/kadmos } \\ \text { RCE } & \text { http://rcenvironment.de } \\ \text { VISTOMS } & \text { https://bitbucket.org/imcovangent/kadmos/src/master/kadmos/vistoms }\end{array}$




\section{References}

[1] Piperni, P., DeBlois, A., and Henderson, R., "Development of a multilevel multidisciplinary-optimization capability for an industrial environment," AIAA journal, Vol. 51, No. 10, 2013, pp. 2335-2352.

[2] Schuhmacher, G., Daoud, F., Petersson, O., and Wagner, M., "Multidisciplinary airframe design optimization," 28th International Congress of the Aeronautical Sciences, 2012.

[3] "Report from ICAS workshop on complex systems integration in aeronautics," 30th ICAS Congress, Daejeon, Korea, 2016.

[4] "Co-Evolution of Aeronautical Complex System \& Complex System Engineering," ICAS Workshop - Complex Systems Integration in Aeronautics, Krakow, 2015.

[5] Ciampa, P. D., and Nagel, B., “Towards the 3rd generation MDO collaboration Environment,” 30th ICAS Congress, Daejeon, Korea, 2016.

[6] Ciampa, P. D., and Nagel, B., "The AGILE Paradigm: the next generation of collaborative MDO," 18th AIAA/ISSMO Multidisciplinary Analysis and Optimization Conference, Denver, USA, 2017.

[7] Bartoli, N., Lefebvre, T., Dubreuil, S., Olivanti, R., Bons, N., Martins, J. R. R. A., Bouhlel, M.-A., and Morlier, J., “An adaptive optimization strategy based on mixture of experts for wing aerodynamic design optimization," 18th AIAA/ISSMO Multidisciplinary Analysis and Optimization Conference, Denver, USA, 2017.

[8] Martins, J. R. R. A., and Lambe, A. B., "Multidisciplinary design optimization: A survey of architectures," AIAA Journal, Vol. 59, No. 9, 2013, pp. 2049-2075.

[9] Tosserams, L., S.and Etman, Papalambors, P., and Rooda, J., “"An augmented lagrangian relaxation for analytical target cascading," Structural and Multidisciplinary Optimization, Vol. 31, No. 3, 2006, pp. 113-122.

[10] Lefebvre, T., Bartoli, N., Dubreuil, S., Panzeri, M., Lombardi, R., Vecchia, P. D., Nicolosi, F., Ciampa, P. D., Anisimov, K., and Savelyev, A., "Methodological enhancements in MDO process investigated in the AGILE European project," 18th AIAA/ISSMO Multidisciplinary Analysis and Optimization Conference, Denver, USA, 2017.

[11] Della Vecchia, P., Stingo, L., Corcione, S., Ciliberti, D., Nicolosi, F., and De Marco, A., "Game theory and evolutionary algorithms applied to MDO in the AGILE European project," AIAA AVIATION, Denver, USA, 2017.

[12] Daoud, F., Maierl, R., Ciampa, P. D., and Gu, X., "Aeroelastic Shape and Sizing Optimization of Aircraft Products supported by AGILE Design Paradigm,” 18th AIAA/ISSMO Multidisciplinary Analysis and Optimization Conference, Denver, USA, 2017.

[13] Lammen, W., de Wit, B., Vankan, J., Timmermans, H., van der Laan, T., and Ciampa, P. D., "Collaborative Design of Aircraft Systems - Multi-Level Optimization of an Aircraft Rudder," 6th CEAS Aerospace Europe Conference, 2017.

[14] Prakasha, P. S., Mirzoyana, A., and Ciampa, P. D., "Collaborative System of Systems Multidisciplinary Design Optimization for Civil Aircraft:AGILE EU project," 18th AIAA/ISSMO Multidisciplinary Analysis and Optimization Conference, Denver, USA, 2017.

[15] Ciampa, P. D., Moerland, E., Seider, D., Baalbergen, E., Lombardi, R., and D’Ippolito, R., “A Collaborative Architecture supporting AGILE Design of Complex Aeronautics Products," 18th AIAA/ISSMO Multidisciplinary Analysis and Optimization Conference, Denver, USA, 2017.

[16] van Gent, I., Ciampa, P. D., Aigner, B., Jepsen, J., Rocca, G. L., and Schut, J., "Knowledge architecture supporting collaborative MDO in the AGILE paradigm," 18th AIAA/ISSMO Multidisciplinary Analysis and Optimization Conference, Denver, USA, 2017.

[17] Haftka, R. T., Villanueva, D., and Chaudhuri, A., "Parallel surrogate-assisted global optimization with expensive functions - a survey," Structural and Multidisciplinary Optimization, 2016, pp. 1-11. doi:10.1007/s00158-016-1432-3, URL http: //dx.doi.org/10.1007/s00158-016-1432-3

[18] Da Ronch, A., Ghoreyshi, M., and Badcock, K., "On the generation of flight dynamics aerodynamic tables by computational fluid dynamics,” Progress in Aerospace Sciences, Vol. 47, No. 8, 2011, pp. 597-620.

[19] Da Ronch, A., Panzeri, M., Abd Bari, M. A., d'Ippolito, R., and Franciolini, M., "Adaptive design of experiments for efficient and accurate estimation of aerodynamic loads," Aircraft Engineering and Aerospace Technology, Vol. 89, No. 4, 2017, pp. $558-569$. 
[20] Zhang, M., Jungo, A., and Bartoli, N., "Disciplinary Data Fusion for Multi-Fidelity Aerodynamic Application,” 6th CEAS Aerospace Europe Conference, Bucharest, Romania, 2017.

[21] Dubreuil, S., Bartoli, N., Gogu, C., and Lefebvre, T., "Efficient global multidisciplinary optimization based on surrogate models," AVIATION/AIAA 2018, 19th AIAA/ISSMO Multidisciplinary Analysis and Optimization Conference, Atlanta, USA, 2018.

[22] Dubreuil, S., Bartoli, N., Gogu, C., and Lefebvre, T., "Propagation of modeling uncertainty by polynomial chaos expansion in multidisciplinary analysis," Journal of Mechanical Design, Vol. 138, No. 11, 2016, p. 111411.

[23] Noesis Solutions, Optimus Rev 10.19 - Users Manual, 2017.

[24] Baalbergen, E., Kos, J., Louriou, C., Campguilhem, C., and Barron, J., "Streamlining cross-organisation product design in aeronautics," Proceedings of the Institution of Mechanical Engineers, Part G: Journal of Aerospace Engineering, 2016, p. 0954410017716480.

[25] Baalbergen, E., Moerland, E., Lammen, W., and Ciampa, P. D., "Methods to Support Efficient Collaboration for Competitive Aircraft Design," 6th CEAS Aerospace Europe Conference, 2017.

[26] van Gent, I., La Rocca, G., and Veldhuis, L. L. M., "Composing MDAO symphonies: graph-based generation and manipulation of large multidisciplinary systems," 18th AIAA/ISSMO Multidisciplinary Analysis and Optimization Conference, Denver, USA, 2017.

[27] Aigner, B., van Gent, I., La Rocca, G., Stumpf, E., and Veldhuis, L. L. M., "Graph-based algorithms and data-driven documents for formulation and visualization of large MDO systems," CEAS Aeronautical Journal, 2018.

[28] van Gent, I., La Rocca, G., and Hoogreef, M. F. M., "CMDOWS: A Proposed New Standard to Store and Exchange MDO Systems," CEAS Aeronautical Journal, 2018.

[29] Lambe, A. B., and Martins, J. R., "Extensions to the design structure matrix for the description of multidisciplinary design, analysis, and optimization processes," Structural and Multidisciplinary Optimization, Vol. 46, No. 2, 2012, pp. $273-284$.

[30] Vankan, J., and Lammen W.F.and Maas, R., "Meta-modeling and multi-objective optimization in aircraft Design," Advances in collaborative civil aeronautical multidisciplinary design optimization. Progress in Astronautics and Aeronautics, $2010, \mathrm{pp}$. 155-192. URL https://doi.org/10.2514/4.867279

[31] Bettebghor, D., Bartoli, N., Grihon, S., Morlier, J., and Samuelides, M., "Surrogate modeling approximation using a mixture of experts based on EM joint estimation," Structural and Multidisciplinary Optimization, Vol. 43, No. 2, 2011, pp. 243-259. URL http://dx.doi.org/10.1007/s00158-010-0554-2, 10.1007/s00158-010-0554-2.

[32] Bouhlel, M. A., Bartoli, N., Otsmane, A., and Morlier, J., "Improving kriging surrogates of high-dimensional design models by Partial Least Squares dimension reduction," Structural and Multidisciplinary Optimization, Vol. 53, No. 5, 2016 , pp. $935-952$. doi:10.1007/s00158-015-1395-9.

[33] Bouhlel, M. A., Bartoli, N., Otsmane, A., and Morlier, J., "An Improved Approach for Estimating the Hyperparameters of the Kriging Model for High-Dimensional Problems through the Partial Least Squares Method," Mathematical Problems in Engineering, Vol. 2016, 2016.

[34] Forrester, A., Keane, A., et al., Engineering design via surrogate modelling: a practical guide, John Wiley \& Sons, 2008.

[35] Lophaven, N., Nielsen, H., and Sondergaard, J., “DACE: A Matlab Kriging Toolbox, Version 2.0, August 1, 2002,” Technical University of Denmark, 2000.

[36] Volkwein, S., "Model reduction using proper orthogonal decomposition," Lecture Notes, Institute of Mathematics and Scientific Computing, University of Graz. see http://www. uni-graz. at/imawww/volkwein/POD. pdf, 2011.

[37] "CPACS - A Common Language for Aircraft Design," http://www. cpacs . de, ????

[38] Powell, M. J., "A direct search optimization method that models the objective and constraint functions by linear interpolation," Advances in optimization and numerical analysis, Springer, 1994, pp. 51-67.

[39] Le Digabel, S., "Algorithm 909: NOMAD: Nonlinear optimization with the MADS algorithm," ACM Transactions on Mathematical Software (TOMS), Vol. 37, No. 4, 2011, p. 44. 
[40] Adams, B. M., Bauman, L. E., Bohnhoff, W. J., Dalbey, K. R., Eddy, J. P., Ebeida, M. S., Eldred, M. S., Hough, P. D., Hu, K. T., Jakeman, J. D., Swiler, L. P., Stephens, J. A., Vigil, D. M., and Wildey., T. M., "Dakota, a multilevel parallel object-oriented framework for design optimization, parameter estimation, uncertainty quantification, and sensitivity analysis: Version 6.1 users manual," Tech. Rep. SAND2014-4633, Sandia National Laboratories, Albuquerque, NM, November 2014. Available online from http://dakota.sandia.gov/documentation.html.

[41] "Noesis Solutions, Optimus - Theoretical Background (available on request),", 2017.

[42] Kennedy, J., and Eberhart, R., "Particle swarm optimization," IEEE International Conference on Neural Networks, 1995 , p. 1942.

[43] Bartoli, N., Kurek, I., Lafage, R., Lefebvre, T., Priem, R., Bouhlel, M.-A., Morlier, J., Stilz, V., and Regis, R., "Improvement of efficient global optimization with mixture of experts: methodology developments and preliminary results in aircraft wing design," 17th AIAA/ISSMO Multidisciplinary Analysis and Optimization Conference, Washington D.C., USA, 2016. doi:10.2514/6.2016-4001.

[44] Bartoli, N., Lefebvre, T., Dubreuil, S., Panzeri, M., D’Ippolito, R., Anisomov, K., and Savelyev, A., "Robust Nacelle Optimization Design investigated in the AGILE European project," AVIATION/AIAA 2018, 19th AIAA/ISSMO Multidisciplinary Analysis and Optimization Conference, Atlanta, United States, 2018.

[45] Ciampa, P. D., Nagel, B., and Consortium, A., "AGILE the next Generation of Collaborative MDO: Achievments and open challenges," AVIATION/AIAA 2018, 19th AIAA/ISSMO Multidisciplinary Analysis and Optimization Conference, Atlanta, United States, 2018. 